The Astrophysical Journal, 669:464-482, 2007 November 1

(C) 2007. The American Astronomical Society. All rights reserved. Printed in U.S.A.

\title{
MOLECULAR OUTFLOWS AND A MID-INFRARED CENSUS OF THE MASSIVE STAR FORMATION REGION ASSOCIATED WITH IRAS 18507+0121
}

\author{
D. S. Shepherd, ${ }^{1}$ M. S. Povich, ${ }^{2}$ B. A. Whitney, ${ }^{3}$ T. P. Robitaille, ${ }^{4}$ D. E. A. Nürnberger,${ }^{5}$ \\ L. Bronfman, ${ }^{6}$ D. P. Stark, ${ }^{7}$ R. Indebetouw, ${ }^{8}$ M. R. Meade, ${ }^{2}$ and B. L. Babler ${ }^{2}$ \\ Received 2007 March 5; accepted 2007 June 28
}

\begin{abstract}
We have observed the central region of the infrared-dark cloud filament associated with IRAS $18507+0121$ at millimeter wavelengths in $\mathrm{CO}(J=1-0),{ }^{13} \mathrm{CO}(J=1-0)$, and $\mathrm{C}^{18} \mathrm{O}(J=1-0)$ line emission and with Spitzer at midinfrared wavelengths. Five massive outflows from two cloud cores were discovered. Three outflows are centered on or near an ultracompact (UC) H II region (G34.4+0.23), while the remaining two outflows originate from the millimeter core G34.4+0.23 MM. Modeling of the spectral energy distributions of the mid-infrared sources identified 31 young stellar objects in the filament with a combined stellar mass of $\sim 127 \pm 27 M_{\odot}$. An additional 22 sources were identified as probable cluster members based on the presence of strong $24 \mu \mathrm{m}$ emission. The total star formation efficiency in the G34.4 cloud filament is estimated to be $\sim 7 \%$, while the massive and intermediate-mass star formation efficiency in the entire cloud filament is estimated to be roughly $2 \%$. A comparison of the gravitational binding energy with the outflow kinetic energy suggests that the compact core containing G34.4+0.23 MM is being destroyed by its molecular outflows, whereas the outflows associated with the more massive core surrounding the G34.4 UC H II region are not likely to totally disrupt the cloud. In addition, a qualitative evaluation of the region appears to suggest that stars in this region may have formed in two stages: first lower mass stars formed and then, a few Myr later, the more massive stars began to form.
\end{abstract}

Subject headings: H II regions — ISM: molecules — stars: formation

\section{INTRODUCTION}

The massive star-forming region associated with IRAS $18507+$ 0121 (hereafter IRAS 18507) is located in an infrared (IR) dark cloud that was mapped by Rathborne et al. (2005) in millimeter and submillimeter continuum and compared to Spitzer mid-IR emission between 3.5 and $8 \mu \mathrm{m}$. IRAS 18507 is roughly $11^{\prime}$ north of the more famous ultracompact (UC) $\mathrm{H}$ II region complex G34.26+0.15 (Wood \& Churchwell 1989; Molinari et al. 1996; Carral \& Welch 1992). The location of the IR-dark cloud relative to G34.26+0.15 is shown in Figure 1.

Near IRAS 18507, Miralles et al. (1994) discovered a UC H II region $(\mathrm{G} 34.4+0.23)$ embedded in a $1000 M_{\odot}$ molecular cloud traced by $\mathrm{NH}_{3}$ emission. The $\mathrm{NH}_{3}$ emission is elongated in the $\mathrm{N}-\mathrm{S}$ direction with a total extent of about $7^{\prime}$; however, the $1.5^{\prime}$ resolution of the observations was not adequate to discern the structure of the core (Miralles et al. 1994). Shepherd et al. (2004, hereafter SNB04) observed IRAS 18507 in $3 \mathrm{~mm}$ continuum emission, $\mathrm{H}^{13} \mathrm{CO}^{+}$and $\mathrm{SiO}$ line emission, and the near-IR. They detected a dense molecular core associated with the UC H II region G34.4+ 0.23 as well as a dense core and embedded protostellar object de-

\footnotetext{
1 National Radio Astronomy Observatory, P.O. Box O, 1003 Lopezville Road, Socorro, NM 87801.

2 Department of Astronomy, University of Wisconsin at Madison, 475 North Charter Street, Madison, WI 53706.

3 Space Science Institute, 4750 Walnut Street Suite 205, Boulder, CO 80301; bwhitney@spacescience.org.

4 Scottish Universities Physics Alliance, School of Physics and Astronomy, University of St Andrews, North Haugh, KY16 9SS, St Andrews, UK; tr9@ st-andrews.ac.uk.

5 European Southern Observatory, Casilla 19001, Santiago 19, Chile.

6 Departamento de Astronomía, Universidad de Chile, Casilla 36-D, Santiago, Chile.

7 Division of Physics, Mathematics, and Astronomy, California Institute of Technology, MS 105-24, Pasadena, CA 91125.

${ }^{8}$ Department of Astronomy, University of Virginia, Charlottesville, VA 22903 0818
}

tected only in millimeter continuum emission, G34.4+0.23 MM (hereafter G34.4 MM). G34.4 MM appears to be a B2 protostar surrounded by $\sim 150 M_{\odot}$ of warm gas and dust. Based on the nondetection of G34.4 MM at near-IR wavelengths, SNB04 suggested the source may be in a very early stage of evolution.

Rathborne et al. (2005) identified four clumps in $1.2 \mathrm{~mm}$ continuum emission which they named MM1-MM4 (see their Fig. 1). MM1 corresponds to G34.4 MM, MM2 is coincident with the UC $\mathrm{H}$ II region G34.4+0.23, MM3 is about $3^{\prime}$ north of G34.4 MM, while MM4 is about $30^{\prime \prime}$ south of the UC H II region.

IRAS 18507 was detected in a $\operatorname{CS}(J=2-1)$ survey of $I R A S$ point sources with far-IR colors suggestive of UC H II regions (Bronfman et al. 1996). The source was selected for further highresolution studies because of its broad-line wings, a signature of current star formation. By modeling $\mathrm{HCO}^{+}, \mathrm{H}^{13} \mathrm{CO}^{+}, \mathrm{CS}$, and $\mathrm{C}^{34} \mathrm{~S}$ spectra obtained at an angular resolution of $\sim 16^{\prime \prime}$, Ramesh et al. (1997) demonstrated that the observed line profiles can be explained by a collapsing hot core of about $800 M_{\odot}$ which is hidden behind a cold $(\sim 4 \mathrm{~K})$ and dense $\left(3 \times 10^{4} \mathrm{~cm}^{-3}\right)$ envelope of about $200 M_{\odot}$.

The kinematic distance to IRAS 18507 has been reported to be between 3.7 and $3.9 \mathrm{kpc}$ based on molecular line velocities that vary from 55 to $59 \mathrm{~km} \mathrm{~s}^{-1}$ depending on the line observed and the position within the cloud (3.9 kpc: Molinari et al. 1996, SNB04, Zhang et al. 2005; 3.8 kpc: Carral \& Welch 1992, Miralles et al. 1994, Ramesh et al. 1997; 3.7 kpc: Rathborne et al. 2005). Kinematic distances can easily have an error of more than $5 \%$ or even $10 \%$ due to observed deviations from circular symmetry and circular motion in our Galaxy. Thus, a difference of $0.2 \mathrm{kpc}$ is easily within the errors for this type of distance determination. Here we adopt the distance of $3.9 \mathrm{kpc}$ to be consistent with Molinari et al. (1996, 1998), SNB04, and Zhang et al. (2005). If the closer distance of $3.7 \mathrm{kpc}$ is more appropriate, then mass estimates derived in this work should be decreased by about $10 \%$, since the mass is proportional to $D^{2}$. 


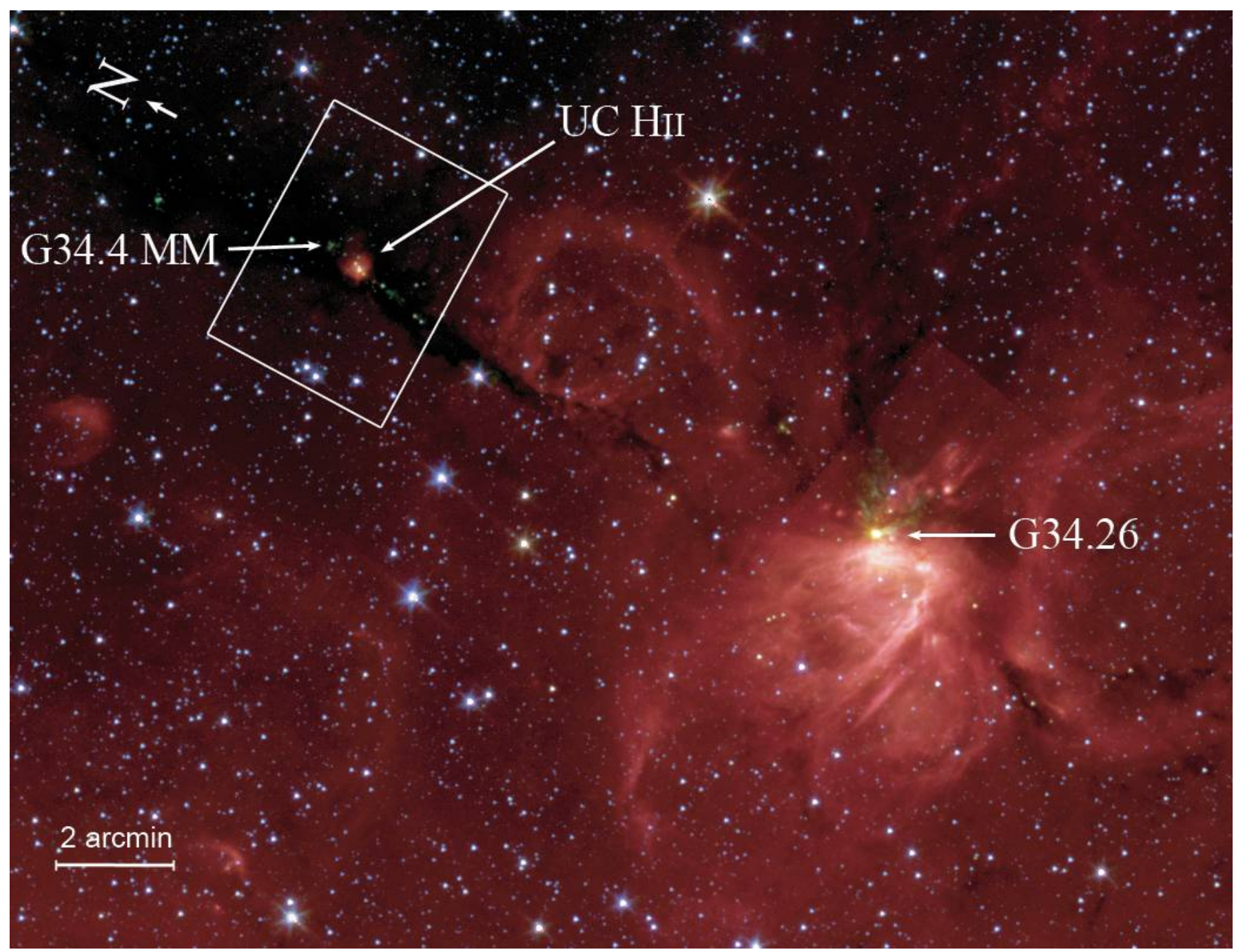

FIG. 1.-Four-color GLIMPSE image showing 3.6, 4.6, 5.8, and $8.0 \mu \mathrm{m}$ as blue, green, orange, and red colors, respectively. The image is displayed in galactic coordinates and is roughly $16.6^{\prime} \times 21.5^{\prime}$ in size. The white box in the top left corner gives the field of view shown in Fig. 2 . The G34.4 UC H II region is in the center of the box within a dense cloud filament seen as a dark lane. The mid-IR counterpart to the G34.4 MM core is seen as a faint green smudge immediately to the upper left of the UC $\mathrm{H}$ II region. The larger nebula in the bottom right corner of the image is associated with the cometary UC H II region G34.26. The bright green emission emanating from this region traces a very energetic outflow. This GLIMPSE image includes the same data as reported in Rathborne et al. (2005), but it covers a wider field of view to show the physical relationship between the IR-dark cloud in which the G34.4 UC H II is located and the H II region G34.26.

Faúndez et al. (2004) mapped the IR-dark cloud with the Swedish-ESO Submillimeter Telescope in $1.2 \mathrm{~mm}$ continuum emission and derived a mass of $2000 M_{\odot}$ in the main condensation based on a dust temperature of $28 \mathrm{~K}$. The total mass of the filament was estimated to be about $3700 M_{\odot}$, where $1000 M_{\odot}$ are associated with G34.4 MM and $2700 M_{\odot}$ are associated with the G34.4 UC H II region (L. Bronfman 2006, private communication). Rathborne et al. (2005) mapped the dark cloud with the IRAM $30 \mathrm{~m}$ telescope in $1.2 \mathrm{~mm}$ continuum emission and derived a cloud mass of $\sim 7500 M_{\odot}$ based on a dust temperature of $34 \mathrm{~K}$, 2 times more mass than derived by Faúndez et al. In this work we assume the temperature and mass estimates of Rathborne et al., since a dust temperature of $34 \mathrm{~K}$ would appear to be more representative of massive star-forming regions. However, a clear reason for why the derived temperature and, hence, mass estimates differ by such a large amount is not clear.

The IRAS 18507 region is also associated with variable $\mathrm{H}_{2} \mathrm{O}$, $\mathrm{CH}_{3} \mathrm{OH}$, and $\mathrm{OH}$ maser emission (Scalise et al. 1989; Palla et al. 1991; Schutte et al. 1993; Miralles et al. 1994; Szymczak et al. 2000; Wang et al. 2006; Edris et al. 2007). Molinari et al. (1996,
1998) observed IRAS 18507 (labeled Mol74 in their papers) and estimated a deconvolved size of the UC H II region of $0.7^{\prime \prime}(0.013 \mathrm{pc}$ at $D=3.9 \mathrm{kpc}$ ).

Based on observations with the NRAO $12 \mathrm{~m}$ telescope, Zhang et al. (2005) report that IRAS 18507 is not associated with molecular outflows. The lack of outflow activity contrasts with the presence of multiple star formation tracers (masers, dense gas, $\mathrm{H}$ II regions, and millimeter continuum).

In this work we present observations of the dense molecular gas near IRAS 18507 at millimeter wavelengths in $\mathrm{CO}(J=1-0)$, ${ }^{13} \mathrm{CO}(J=1-0)$, and $\mathrm{C}^{18} \mathrm{O}(J=1-0)$ line emission to map the dense gas morphology and determine if there are massive outflows associated with IRAS 18507. Archive data from the Spitzer GLIMPSE survey at mid-IR wavelengths were studied to obtain a census of the massive and intermediate-mass star cluster properties over the entire IR-dark cloud filament. Section 2 gives an

9 The National Radio Astronomy Observatory is a facility of the National Science Foundation operated under cooperative agreement by Associated Universities, Inc. 
overview of the observations, $\S 3$ presents the results, and $\S 4$ discusses implications.

\section{OBSERVATIONS}

\subsection{Observations in the $3 \mathrm{~mm}$ Band}

Simultaneous observations in $\mathrm{CO}(J=1-0),{ }^{13} \mathrm{CO}(J=1-0)$, and $\mathrm{C}^{18} \mathrm{O}(J=1-0)$ lines were made with the Owens Valley Radio Observatory (OVRO) array of six $10.4 \mathrm{~m}$ telescopes on 2004 March 27 and 29 and 2004 April 16 and 18. Projected baselines ranging from about 12 to $115 \mathrm{~m}$ provided sensitivity to structures up to about $20^{\prime \prime}$ with $\sim 5^{\prime \prime}$ resolution. Two fields were observed as a Nyquist-sampled mosaic. The observations were alternated between fields every 9 minutes to assure that the $u v$ coverage in each field was similar. The total integration time on each field was approximately $8.8 \mathrm{hr}$. Cryogenically cooled SIS receivers operating at $4 \mathrm{~K}$ produced typical single sideband system temperatures of about $400 \mathrm{~K}$. The gain calibrator was the quasar $1751+096$, and the passband calibrators were $3 \mathrm{C} 345,3 \mathrm{C} 454.3$, and $3 \mathrm{C} 273$. Observations of Uranus provided the flux density calibration scale with an estimated uncertainty of $\sim 15 \%$. Calibration was carried out using the Caltech MMA data reduction package (Scoville et al. 1993). Images were produced using the MIRIAD software package (Sault et al. 1995) and deconvolved with a maximum entropy (MEM) algorithm. Data analysis was performed using both the MIRIAD package and the Common Astronomy Software Applications package.

The spectral bandpass for each line was centered on a velocity of $57.0 \mathrm{~km} \mathrm{~s}^{-1}$. The $\mathrm{CO}$ images have a synthesized beam of $3.83^{\prime \prime} \times 3.45^{\prime \prime}(\mathrm{FWHM})$ at P.A. $-59.7^{\circ}$, spectral resolution $1.3 \mathrm{~km} \mathrm{~s}^{-1}$, and rms noise $60 \mathrm{mJy}^{-1}$ beam ${ }^{-1}$. The ${ }^{13} \mathrm{CO}$ images have a synthesized beam of $4.04^{\prime \prime} \times 3.83^{\prime \prime}(\mathrm{FWHM})$ at P.A. $-49.9^{\circ}$, spectral resolution $1.36 \mathrm{~km} \mathrm{~s}^{-1}$, and rms noise $44.0 \mathrm{mJy}^{\text {beam }}{ }^{-1}$. The $\mathrm{C}^{18} \mathrm{O}$ images have a synthesized beam of $4.04^{\prime \prime} \times 3.83^{\prime \prime}$ (FWHM) at P.A. $-50.1^{\circ}$, spectral resolution $0.683 \mathrm{~km} \mathrm{~s}^{-1}$, and rms noise $56.0 \mathrm{mJy}^{-1}$ beam $^{-1}$.

\subsection{Spitzer Images}

Mid-IR images at 3.6, 4.6, 5.8, and $8.0 \mu \mathrm{m}$ were obtained using the Infrared Array Camera (IRAC; Fazio et al. 2004) of Spitzer as part of the Galactic Legacy Infrared Mid-Plane Survey Extraordinaire (GLIMPSE; Benjamin et al. 2003). Image mosaics were created by the GLIMPSE pipeline after image artifacts such as cosmic rays, stray light, column pull-down, and banding were removed. ${ }^{10}$ Point sources were extracted from the GLIMPSE images using a modified version of Daophot (Stetson 1987). The astrometric uncertainty for point sources is $\sim 0.3^{\prime \prime}$. The GLIMPSE $5 \sigma$ point-source sensitivities are approximately $0.3,0.3,1.2$, and $0.7 \mathrm{mJy}$ in the four IRAC bandpasses, respectively. Point-source sensitivities were generally higher in areas of bright diffuse background emission. The fluxes of point sources that are detected at $\geq 5 \sigma$ twice in one IRAC band or once in each of two bands are entered in the GLIMPSE point-source archive. Each archive entry includes the corresponding $J, H$, and $K_{s}$ fluxes from the 2MASS point-source catalog (Skrutskie et al. 2006) if that source was detected in 2MASS.

The multiband imaging photometer for Spitzer (MIPS; Rieke et al. 2004) was used to image the entire GLIMPSE survey area at 24 and $70 \mu \mathrm{m}$ as part of the MIPSGAL survey (Carey et al. 2005). Fluxes at $24 \mu \mathrm{m}$ for point sources within the G34.4 IR-dark cloud were extracted from the frame using the GLIMPSE point-

\footnotetext{
${ }^{10}$ Details of the data processing can be found at http://www.astro.wisc.edu/ glimpse/docs.html.
}

source extractor. The relative uncertainty between two sources in a MIPSGAL image is roughly $0.3-0.5$ pixels, while the absolute positional uncertainty is $\sim 1^{\prime \prime}$.

\section{RESULTS}

Figure 1 presents a four-color $(3.6,4.6,5.8$, and $8.0 \mu \mathrm{m})$ GLIMPSE image of the G34.4 environment in Galactic coordinates (north points to the top left corner). The "cometary" UC $\mathrm{H}$ II region G34.26+0.15 is located on the right side of the image. An IR-bright nebula extends below the G34.26 UC H II region. The UC H II region contains a cluster of OB stars, the most luminous of which is an O6.5 spectral type (Wood \& Churchwell 1989). The nebula is bright in the IRAC bands primarily due to PAH emission features stimulated by UV radiation from the cluster. The subject of this paper is the dark filament in the top left which is forming early B stars. The mid-IR emission from the UC $\mathrm{H}$ II region G34.4+0.23 is significantly less than that of G34.26, as expected due to the lower luminosity of the central source. Figure 2 presents a close-up of the IR-dark filament, now displayed in equatorial coordinates with north pointing upward. Along the dark filament, north and south of G34.4, are embedded sources seen predominantly by their $4.5 \mu \mathrm{m}$ (green) emission. Our OVRO CO mosaic is centered on the southern core near the $\mathrm{UC} \mathrm{H}$ II region G34.4 and the millimeter core G34.4 MM located $\sim 40^{\prime \prime}$ north of the $\mathrm{UC} \mathrm{H}$ II region.

Figure 3 presents the red- and blueshifted emission near G34.4 MM and G34.4 along with a $2 \mu \mathrm{m} K$-band image from $\mathrm{SNB} 04$. Neither the millimeter core nor the $\mathrm{UC} \mathrm{H}$ II region is visible in the $K$-band image. Both regions show complex high-velocity structure, suggesting the presence of multiple outflows.

Figure 4 shows the integrated emission (moment 0 ) in $\mathrm{C}^{18} \mathrm{O}$ and ${ }^{13} \mathrm{CO}$ along with the red- and blueshifted ${ }^{13} \mathrm{CO}$ emission. The bottom two panels in Figure 4 show moment 1 maps of $\mathrm{C}^{18} \mathrm{O}$ and ${ }^{13} \mathrm{CO}$ in which the brightness of the color is proportional to the intensity in the moment 0 image. This type of image provides some insight into how the dense cloud structure and kinematics are being affected by the molecular outflows. Figures 5, 6, and 7 present channels maps of $\mathrm{CO},{ }^{13} \mathrm{CO}$, and $\mathrm{C}^{18} \mathrm{O}$ emission, respectively.

\subsection{Molecular Outflows}

At least two outflows can be seen emanating from G34.4 MM (labeled A and B in Fig. 3, top left), while at least three outflows originate from the southern core near the G34.4 UC H II region (labeled C, D, and E).

The velocity structure of the outflows from the G34.4 MM region is consistent with a single outflow dominating the redshifted kinematics (outflow G34.4:A). There is a weak blueshifted counter flow extending to the northeast that can be traced to G34.4 MM. The redshifted lobe of outflow G34.4:A appears to have little or no associated blueshifted emission, suggesting that it is oriented outside of the plane of the sky (the blueshifted emission in the red lobe appears to be from the collimated flow G34.4:E coming from the southern region). The full opening angle of the redshifted lobe is $\sim 43^{\circ}$.

Marginal evidence for an outflow perpendicular to outflow G34.4:A is seen in the red- and blueshifted emission clumps along the axis labeled B. No source has been detected at millimeter wavelengths which could drive outflow G34.4:B. The source could be embedded in G34.4 MM and not resolved at the resolution of $\sim 5^{\prime \prime}$ of SNB04.

The southern molecular core containing the G34.4 UC H II region contains three overlapping flows seen in the $\mathrm{CO}$ emission (flows $\mathrm{C}, \mathrm{D}$, and $\mathrm{E}$ ). Blueshifted $\mathrm{CO},{ }^{13} \mathrm{CO}$, and $\mathrm{C}^{18} \mathrm{O}$ gas is seen 

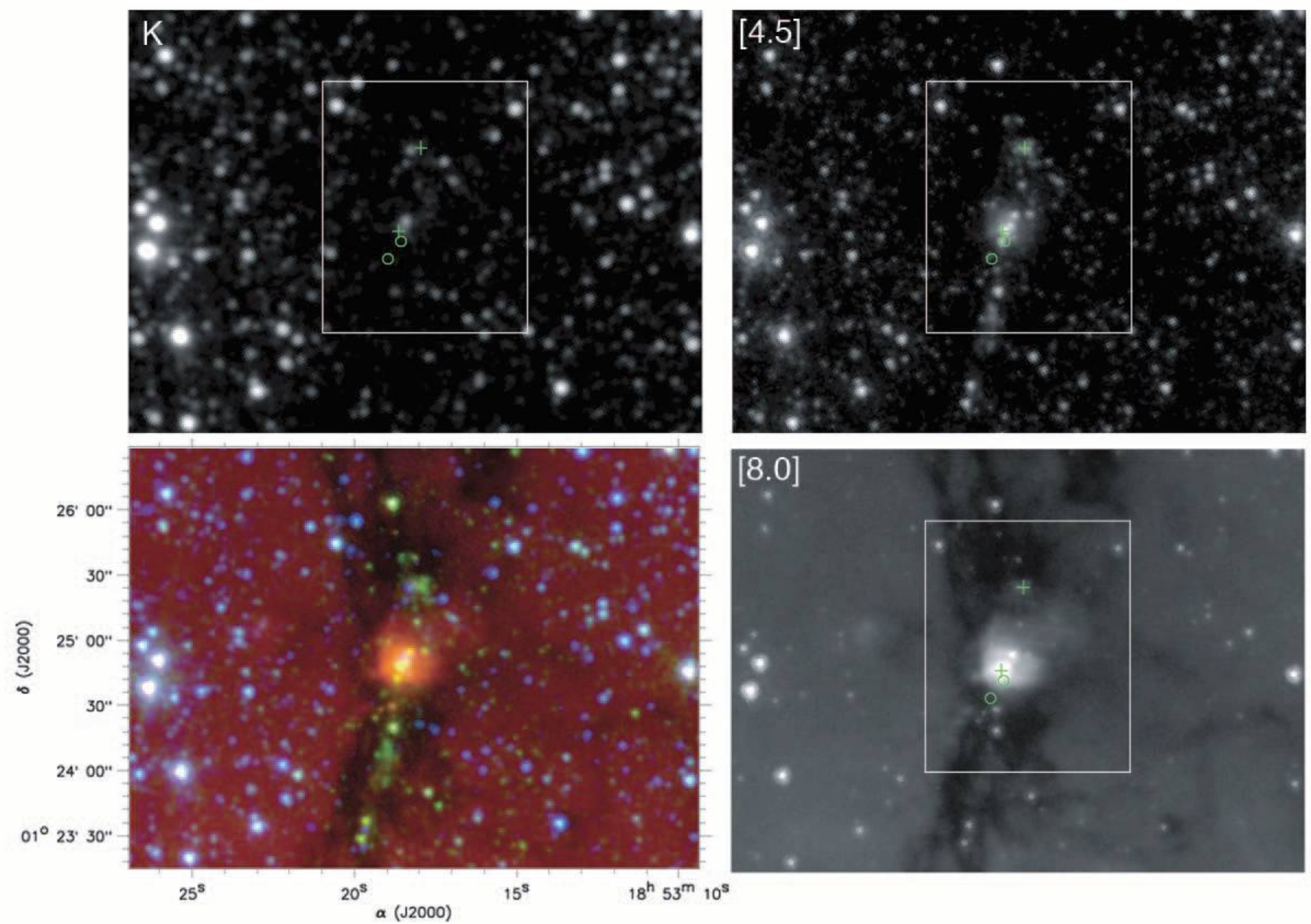

FIG. 2.-Four images, all showing the same field of view. Clockwise from the top left panel are: (a) 2MASS $K$ band, (b) GLIMPSE 4.5 $\mu \mathrm{m}$, (c) GLIMPSE 8.0 $\mu \mathrm{m}$, and $(d)$ a three-color image combining the three other images as blue, green, and red, respectively. Green plus signs mark the positions of the UC H II and G34.4 MM, while green circles show the locations predicted for the driving sources of outflows G34.4:D and G34.4:E. The white box gives the approximate field of view of Fig. 3. The locations of the millimeter cores detected by Rathborne et al. (2005) in J2000.0 coordinates are: MM1: $18^{\mathrm{h}} 53^{\mathrm{m}} 18.0^{\mathrm{s}}, 01^{\circ} 25^{\prime} 24^{\prime \prime} ; \mathrm{MM}^{\prime}: 18^{\mathrm{h}} 53^{\mathrm{m}} 20.4^{\mathrm{s}}, 01^{\circ} 28^{\prime} 23^{\prime \prime}$; and MM4: $18^{\mathrm{h}} 53^{\mathrm{m}} 19.0^{\mathrm{s}}, 01^{\circ} 24^{\prime} 08^{\prime \prime}$.

toward the G34.4 UC H il region extending to the west (flow G34.4:C). This outflow is seen most clearly in ${ }^{13} \mathrm{CO}$ and $\mathrm{C}^{18} \mathrm{O}$ emission in Figure 4. ${ }^{13} \mathrm{CO}$ blueshifted emission is traced back to the $\mathrm{H}$ II region (Fig. 4, top right), while the bottom two panels of Figure 4 illustrating the velocity structure in the dense gas show that blueshifted emission extends from the $\mathrm{UC} \mathrm{H}$ II region to the west. A possible redshifted outflow lobe east of the UC H II may be visible in ${ }^{13} \mathrm{CO}$ emission in the top right panel in Figure 4 although the identification is not definitive.

The northeast-southwest flow (G34.4:D) appears to be centered about $10^{\prime \prime}$ southeast of the UC $\mathrm{H}$ II region, just off the peak of a dense $\mathrm{C}^{18} \mathrm{O}$ clump. The flow appears to be relatively symmetric in the red- and blueshifted gas distribution although the actual morphology is considerably confused due to overlapping outflows. If the flow is symmetric, then one would expect the driving source to be located near the small, filled triangle at $18^{\mathrm{h}} 53^{\mathrm{m}} 19.0^{\mathrm{s}}, 01^{\circ} 24^{\prime} 35^{\prime \prime}$ along the G34.4:D outflow axis in Figures 3 and 4.

The southeast-northwest flow (G34.4:E) is seen clearly in both $\mathrm{CO}$ and ${ }^{13} \mathrm{CO}$ emission. If the driving source is located between the red-and blueshifted emission along the outflow axis, it would be centered about $5^{\prime \prime}$ south-southwest of the UC H II region (indicated by a small, filled triangle at $18^{\mathrm{h}} 53^{\mathrm{m}} 18.6^{\mathrm{s}}, 01^{\circ} 24^{\prime} 43^{\prime \prime}$ along the G34.4:E outflow axis in Figs. 3 and 4). The outflow is not symmetric: the redshifted emission lobe extends about $20^{\prime \prime}$ to the southeast, while the blueshifted lobe extends roughly $60^{\prime \prime}$ to the northwest, overlapping the blueshifted lobe from the G34.4 UC $\mathrm{H}$ II region flow and the redshifted lobe of flow G34.4:A farther north.

Outflow G34.4:A has a position angle of $+58^{\circ}$ (measured from north to the axis of the blueshifted lobe), while for G34.4:B P.A. = $-38^{\circ}$ and the difference in orientation between outflow axes is $96^{\circ}$. The position angles of the three outflows near the G34.4 UC H II region are P.A. $=-100^{\circ}, 38^{\circ}$, and $-34^{\circ}$ for outflows G34.4:C, G34.4:D, and G34.4:E, respectively, and the maximum difference in orientation between the axes is $138^{\circ}$.

\subsection{Mass and Kinematics of the Outflows}

The mass associated with $\mathrm{CO}$ line emission is calculated following Scoville et al. (1986). The CO excitation temperature is taken to be $34 \mathrm{~K}$, which is the dust temperature in MM1 derived from fits to the spectral energy distribution (SED; Rathborne et al. 2005). We assume the gas is in LTE with $[\mathrm{CO}] /\left[\mathrm{H}_{2}\right]=10^{-4}$, $[\mathrm{CO}] /\left[{ }^{13} \mathrm{CO}\right]=51$, and $[\mathrm{CO}] /\left[\mathrm{C}^{18} \mathrm{O}\right]=378$ at the Galactocentric distance of $5.8 \mathrm{kpc}$ (Wilson \& Rood 1994).

The $\mathrm{CO}$ optical depth at red- and blueshifted velocities is estimated from the $\mathrm{CO} /{ }^{13} \mathrm{CO}$ ratio of spectra taken at four positions in the mosaic image that have been convolved with a $10^{\prime \prime}$ beam. The open triangles in Figure 4 (top middle) show the locations 


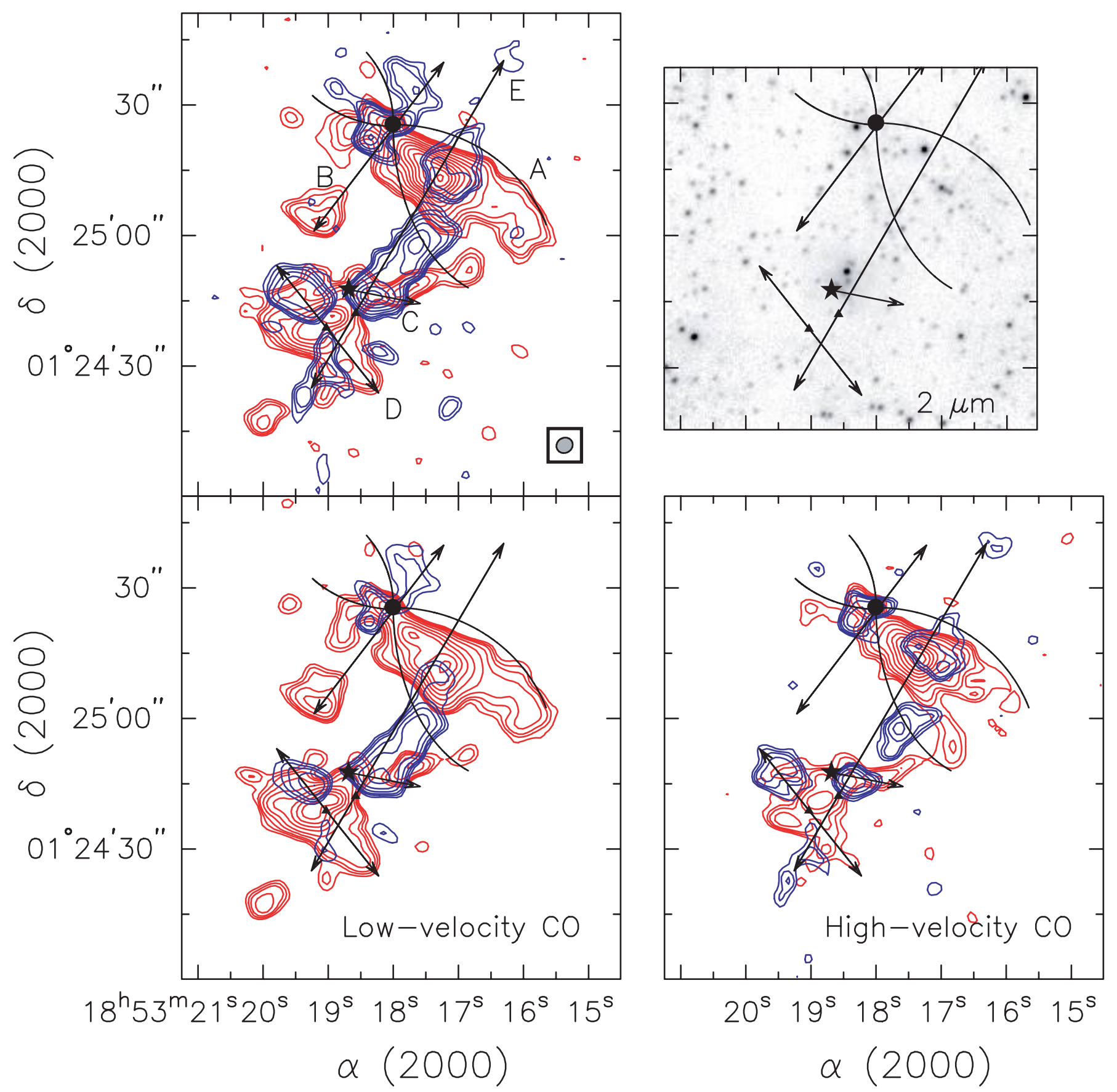

Fig. 3. - $\operatorname{CO}(J=1-0)$ red- and blueshifted emission. Top left: Red- and blueshifted emission from 62.3 to $79.2 \mathrm{~km} \mathrm{~s}^{-1}$ and 37.5 to $53.2 \mathrm{~km} \mathrm{~s}^{-1}$, respectively. The rms is $470 \mathrm{mJy}_{\text {beam }}^{-1} \mathrm{~km} \mathrm{~s}^{-1}$ in the redshifted image and $370 \mathrm{mJy} \mathrm{beam}^{-1} \mathrm{~km} \mathrm{~s}^{-1}$ in the blueshifted image. Bottom: Low-velocity CO emission (left; $62.3-67.5 \mathrm{~km} \mathrm{~s}^{-1}$ and $49.3-53.2 \mathrm{~km} \mathrm{~s}^{-1}, \mathrm{rms}=350 \mathrm{mJy}_{\text {beam }}^{-1} \mathrm{~km} \mathrm{~s}^{-1}$ ) and higher velocity CO emission $\left(r i g h t ; 67.5-79.2 \mathrm{~km} \mathrm{~s}^{-1}\right.$ and $\left.37.5-49.3 \mathrm{~km} \mathrm{~s}^{-1}, \mathrm{rms}^{2}=350 \mathrm{mJy} \mathrm{beam}^{-1} \mathrm{~km} \mathrm{~s}^{-1}\right)$. In all contour maps, contours are plotted at 3,4,5,7,10,15, and $20 \sigma$ and continue with a spacing of $5 \sigma$. Top right: Near-IR $K$-band image of SNB04 with the locations of the proposed outflows overlaid. In all panels, the solid circles represent the location of G34.4 MM, the stars show the location of the UC $\mathrm{H}$ II region, and the outflow (A) from G34.4 MM is outlined with a series of arcs which delineate the approximate boundaries of the flow. Four other proposed outflows are shown as arrows. A solid triangle indicates the possible location of the driving source of an outflow. The outflow from the UC H II region is one-sided, since only the blueshifted outflow is clearly detected. The synthesized beam of $3.83^{\prime \prime} \times 3.45^{\prime \prime}$ at P.A. $=-59.7^{\circ}$ is shown in the bottom right corner of the top left panel.

where the spectra were taken, while Figure 8 shows the spectra taken at those positions. In channels where no ${ }^{13} \mathrm{CO}$ is detected, we assume that the $\mathrm{CO}$ is optically thin. The $\mathrm{CO}$ and ${ }^{13} \mathrm{CO}$ line profiles show a dip near $v_{\text {LSR }}$, indicating that the lines suffer from selfabsorption and/or the interferometer is missing extended flux at lower velocities.

Because multiple, overlapping flows are present, it is not possible to obtain a reasonable estimate of the inclination of each flow. Thus, we assume an inclination angle of $45^{\circ}$, which minimizes errors introduced by inclination effects for flows that are not near the plane of the sky or along the line of sight. Table 1 summarizes the physical properties of the molecular gas in the combined outflows originating from $\mathrm{G} 34.4 \mathrm{MM}$ and those centered near the $\mathrm{UC} H$ II region. The total flow mass $M_{f}$ is given by $\sum M_{i}$, where $M_{i}$ is the flow mass in velocity channel $i$ corrected for optical depth. The momentum $P$ is given by $\sum M_{i} v_{i}$ and the kinetic energy $E$ by $\frac{1}{2} \sum M_{i} v_{i}^{2}$, where $v_{i}$ is the central velocity of the channel relative to $v_{\mathrm{LSR}}$. 

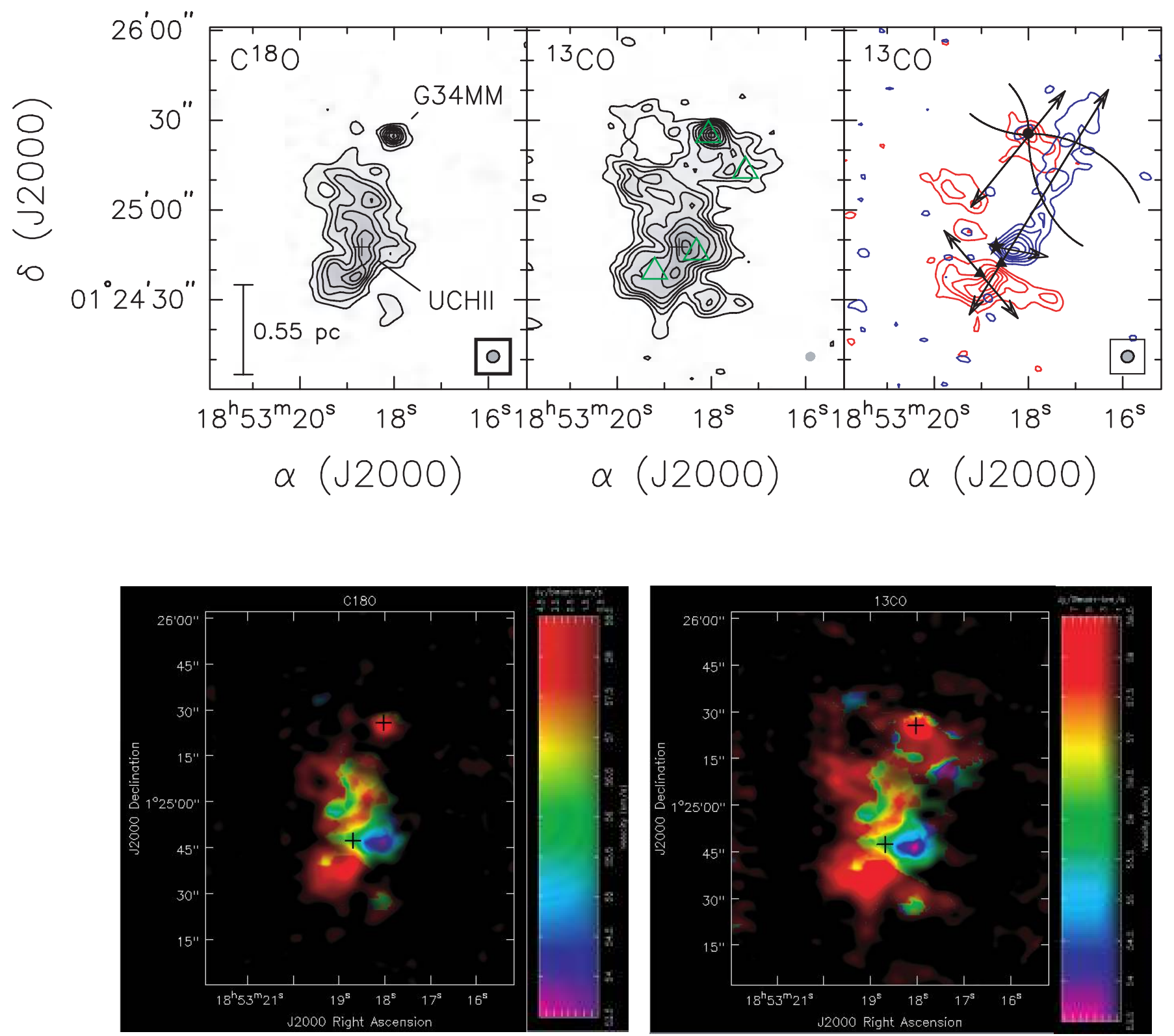

FIG. 4. $-\mathrm{C}^{18} \mathrm{O}$ (top left) and ${ }^{13} \mathrm{CO}$ (top middle) integrated emission (moment 0 ) from 62.1 to $54.6 \mathrm{~km} \mathrm{~s}^{-1}$ and 64.5 to $49.5 \mathrm{~km} \mathrm{~s}^{-1}$, respectively $\left(v_{\mathrm{LSR}}=57 \mathrm{~km} \mathrm{~s}^{-1}\right.$ ). The $\mathrm{rms}$ in the $\mathrm{C}^{18} \mathrm{O}$ image is $200 \mathrm{mJy}$ beam ${ }^{-1} \mathrm{~km} \mathrm{~s}^{-1}$; contours are plotted at $3,5,7,9,11,13,15$, and $17 \sigma$, while gray scale is shown from $200 \mathrm{mJy} \mathrm{beam}^{-1} \mathrm{~km} \mathrm{~s}^{-1}$ to a peak of $3.1 \mathrm{Jy}$ beam ${ }^{-1} \mathrm{~km} \mathrm{~s}^{-1}$. The rms in the ${ }^{13} \mathrm{CO}$ image is $280 \mathrm{mJy}_{\text {beam }}^{-1} \mathrm{~km} \mathrm{~s}^{-1}$; contours are plotted at 3, 5, 7, 9, 12, 15, 20, 25, and $30 \sigma$, while gray scale is shown from $280 \mathrm{mJy}_{\text {beam }}{ }^{-1} \mathrm{~km} \mathrm{~s}^{-1}$ to a peak of $9.0 \mathrm{Jy}$ beam ${ }^{-1} \mathrm{~km} \mathrm{~s}^{-1}$. Plus symbols represent the locations of the UC H II region and G34.4 MM. Green triangles represent the locations of spectra taken for optical depth estimates in the outflows. A scale size of 0.55 is shown in the left panel; synthesized beams are shown in the bottom right of each panel. The top right panel shows ${ }^{13} \mathrm{CO}$ red- and blueshifted emission from 63.1 to $61.8 \mathrm{~km} \mathrm{~s}^{-1}$ and 52.3 to $49.5 \mathrm{~km} \mathrm{~s}^{-1}$, respectively. The rms in the image is $100 \mathrm{mJy}$ beam ${ }^{-1} \mathrm{~km} \mathrm{~s}^{-1}$. Contours are plotted at $3,6,9,12,15,18$, and $21 \sigma$. Symbols are the same as in Fig. 3. Bottom: Moment 1 maps of $\mathrm{C}^{18} \mathrm{O}$ and ${ }^{13} \mathrm{CO}$ in which the brightness of the color is proportional to the intensity in the moment 0 image. Color bars show velocity and brightness scales in each image.

The dynamical timescale $t_{d}$ is calculated using $R_{f} /\langle V\rangle$, where the intensity-weighted velocity $\langle V\rangle$ is given by $P /\left(\sum M_{i}\right)$ (Cabrit $\&$ Bertout 1990) and $R_{f}$ is the average outflow radius of the flows. This method of calculating $t_{d}$ is really only correct for a single outflow; however, an independent outflow age cannot be determined for both flows in the cores, since they cannot be separated near the central position. Because of this uncertainty, the flow dynamical timescale could easily be in error by a factor of 2-5.

The mass outflow rate $\dot{M}_{f}$ is $\sum M_{i} / t_{d}$, the force $F$ is $P / t_{d}$, and the mechanical luminosity $L$ is $E / t_{d}$. Since these values are dependent on $t_{d}^{-1}$, uncertainties in $t_{d}$ are directly applicable to $\dot{M}_{f}, F$, and $L$.

The combined outflows from the G34.4 core have a total mass of $111 M_{\odot}$ and energy $E=7.8 \times 10^{46} \mathrm{ergs}$. The G34.4 MM out- flows have a combined mass of $34.8 M_{\odot}$ and energy $E=4.9 \times$ $10^{46}$ ergs. Despite the uncertainties, the flow masses and energies are consistent with those for outflows driven by young, early B stars.

\subsection{The Dense Gas Cores}

The $\mathrm{C}^{18} \mathrm{O}$ and ${ }^{13} \mathrm{CO}$ moment 0 images (Fig. 4) show clearly that the two cores have very different characteristics. The southern core centered on the G34.4 UC H II region is extended, with a largest spatial scale of about $1.4 \mathrm{pc}$ at a distance of $3.9 \mathrm{kpc}$. The northern core, centered on G34.4 MM, is significantly more compact with higher peak emission than the southern cloud. The diameter of the G34.4 MM core is about $0.2 \mathrm{pc}$ and is surrounded by faint, diffuse ${ }^{13} \mathrm{CO}$ emission that extends out to about $1 \mathrm{pc}$ in size. 


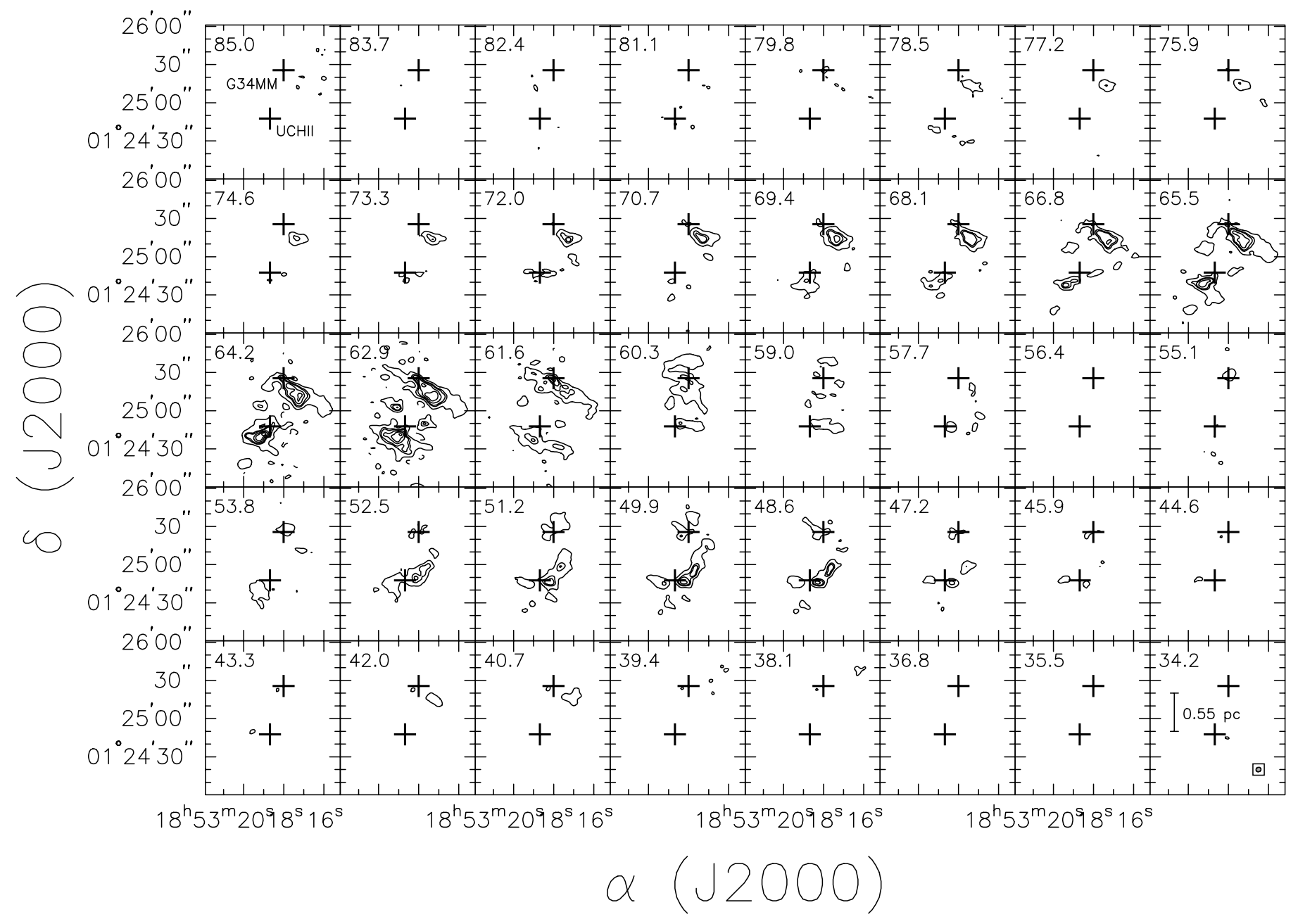

FIG. 5.- $\mathrm{CO}(J=1-0)$ channel maps at $1.3 \mathrm{~km} \mathrm{~s}^{-1}$ spectral resolution. The central velocity is indicated in the top left corner of each panel. The channel rms is $60 \mathrm{mJy}$ beam ${ }^{-1}$, the peak is $3.3 \mathrm{Jy} \mathrm{beam}^{-1}$, and contours are plotted at $\pm 5,15,20,30,40$, and $50 \sigma$. The bottom right panel shows the synthesized beam in the bottom right corner $\left(3.83^{\prime \prime} \times 3.45^{\prime \prime}\right.$ at P.A. $\left.=-59.7^{\circ}\right)$ and a scale size of $0.55 \mathrm{pc}$. Plus symbols represent the locations of the UC $\mathrm{H}$ II region at the $6 \mathrm{~cm}$ continuum peak and G34.4 MM as indicated in the top left panel.

The total mass of the 2 cores as measured from $\mathrm{C}^{18} \mathrm{O}$ is given in Table 2 . $\mathrm{C}^{18} \mathrm{O}$ is assumed to be optically thin although this is not likely to be true near $v_{\mathrm{LSR}}$ at the peak of the line profile. The extended, southern cloud in which the UC H II region is embedded is almost 10 times more massive $\left(692 M_{\odot}\right)$ than the G34.4 MM core $\left(75 M_{\odot}\right)$.

As Figure 8 illustrates, the $\mathrm{C}^{18} \mathrm{O}$ line appears to be suffering from missing flux due to the lack of short baselines. This is most apparent in the spectra taken southeast of the UC H II region position where $\mathrm{C}^{18} \mathrm{O}$ is more extended and there is a clear doublepeaked line profile. The $\mathrm{C}^{18} \mathrm{O}$ line is single peaked closer to the cloud core, suggesting that the interferometer is recovering most of the flux density and we are not seeing significant self-absorption. Thus, the mass estimates based on $\mathrm{C}^{18} \mathrm{O}$ are lower limits.

The G34.4 and G34.4 MM molecular cores have a projected separation of about $40^{\prime \prime}(0.75 \mathrm{pc}$ at $3.9 \mathrm{kpc})$. Their LSR velocities as measured by the peak of the $\mathrm{C}^{18} \mathrm{O}$ line differ by $1.4 \mathrm{~km} \mathrm{~s}^{-1}$.

\subsection{Mid-IR Sources: GLIMPSE AND MIPSGAL}

G34.4 MM and the G34.4 UC H II region are embedded in an extended, filamentary IR-dark cloud complex seen against the diffuse PAH background emission which is visible primarily in the Spitzer $8.0 \mu \mathrm{m}$ band (Figs. 1 and 2). The G34.4 MM core is associated with diffuse emission in all four IRAC bands, but is par- ticularly noticeable at $4.5 \mu \mathrm{m}$. The [4.5] band contains the $\mathrm{H}$ I $\operatorname{Br} \alpha$ line along with some molecular forbidden lines, notably the $\operatorname{CO}(J=1-0) \mathrm{P}(8)$ fundamental band head, but unlike the other three bands, does not contain any PAH emission feature. The [4.5] band also contains several $\mathrm{H}_{2}$ lines [e.g., the $\mathrm{H}_{2}(J=0-0) \mathrm{S}(9)$ line at $4.694 \mu \mathrm{m}]$ which trace shocks in some massive outflows (e.g., DR 21: Smith et al. 2006; W75N: Davis et al. 2007). Thus, the [4.5] band appears to readily trace ionized and/or shocked gas.

Figure 9 shows the IRAC $4.5 \mu \mathrm{m}$ image in gray scale with ${ }^{13} \mathrm{CO}$ contours overlaid for reference. The plus symbols represent the locations of the $\mathrm{UC} \mathrm{H}$ II region and the G34.4 MM core. The diamonds just south and southeast of the UC H II region represent the predicted locations of sources driving outflows G34.4: E $\left(18^{\mathrm{h}} 53^{\mathrm{m}} 18.6^{\mathrm{s}}, 01^{\circ} 24^{\prime} 43^{\prime \prime}\right)$ and G34.4:D $\left(18^{\mathrm{h}} 53^{\mathrm{m}} 19.0^{\mathrm{s}}\right.$, $01^{\circ} 24^{\prime} 35^{\prime \prime}$ ) based on $\mathrm{CO}$ and ${ }^{13} \mathrm{CO}$ emission. While the predicted position of the YSO that was identified based on the $\mathrm{CO}$ outflow morphology should be considered as being no better than an educated guess, it is interesting that each position is associated with a mid-IR source to within a few arcseconds. The characteristics of these sources along with other sources in the cluster are discussed below.

The SEDs were analyzed by fitting model SEDs to each observed source from a large precomputed grid of YSO models (Robitaille et al. 2006, 2007). The grid consists of 20,000 two-dimensional 


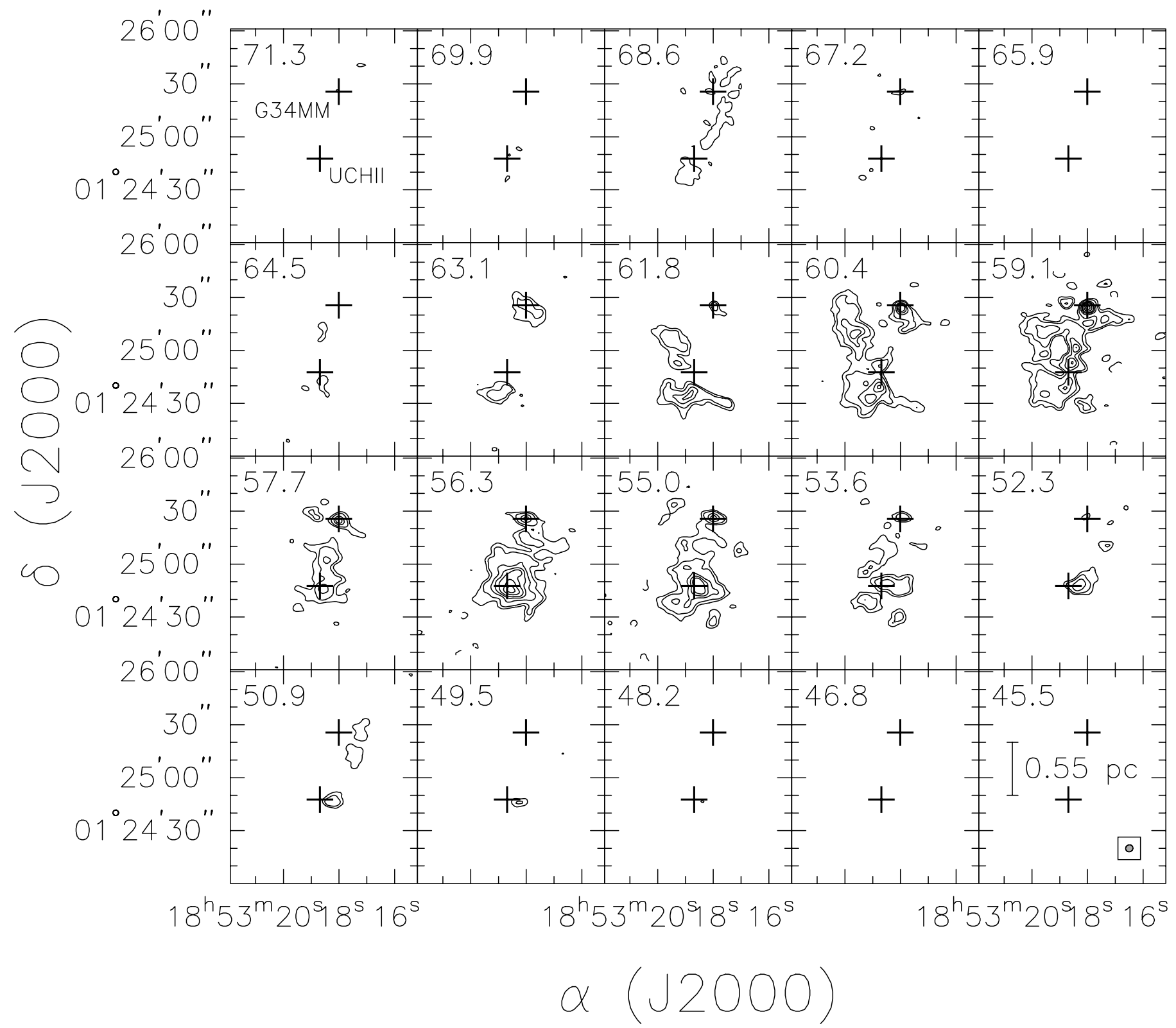

FIG. 6. $-{ }^{13} \mathrm{CO}(J=1-0)$ channel maps at $1.36 \mathrm{~km} \mathrm{~s}^{-1}$ spectral resolution. The central velocity is indicated in the top left corner of each panel. The channel $\mathrm{rms}$ is $44 \mathrm{mJy}_{\text {beam }}{ }^{-1}$, the peak is $1.7 \mathrm{Jy}_{\text {beam }}{ }^{-1}$, and contours are plotted at $\pm 3,5,7,9,12,15,20$, and $25 \sigma$. The bottom right panel shows the synthesized beam in the bottom right corner $\left(4.04^{\prime \prime} \times 3.83^{\prime \prime}\right.$ at P.A. $\left.=-49.9^{\circ}\right)$ and a scale size of $0.55 \mathrm{pc}$. Plus symbols represent the locations of the UC H In region at the $6 \mathrm{~cm}$ continuum peak and G34.4 MM as indicated in the top left panel.

YSO radiation transfer models spanning a complete range of stellar mass and evolutionary stage. Each YSO model outputs SEDs at 10 viewing angles (inclinations), so the fitter actually has 200,000 SEDs to choose from. The YSO models also output SEDs for each of 50 aperture sizes. Thus, if the distance to the star formation region is known, only the model SEDs with the appropriate aperture size compared to the observed photometry are used. A grid of stellar atmospheres were also incorporated into the fitting algorithm in order to facilitate the separation of main-sequence and evolved stars from YSOs (Brott \& Hauschildt 2005; Kurucz 1993). The fitting algorithm includes a foreground extinction component to the model which allows one to easily distinguish highly reddened stars from YSOs which have an additional emission component in the mid-IR.

The $24 \mu \mathrm{m}$ fluxes were cross-correlated with positions of the 2199 GLIMPSE archive sources lying within a 3.9' radius of the
G34.4 UC H II region to account for observational errors in addition to photon counting statistics. For the purposes of the fitting, all flux uncertainties that fell below the $10 \%$ level were reset to a minimum value of $10 \%$. The SED fitter was then run on this combined eight-band source list, fitting only the fluxes of sources detected in four or more bands. Sources with SEDs well fit by a stellar atmosphere are not likely to be cluster members. Sources with a $\chi^{2}$ per flux data point $\left(\chi^{2} / N_{\text {data }}\right)$ of 4.8 provided a reasonable dividing line between sources that, by eye, appeared to be well fit by stellar atmospheres and those that were not well fit due to IR excesses. Of the total 2199 sources in the GLIMPSE archive in this region, 868 were detected in four or more bands. Of those, 829 sources were well fit by stellar atmospheres and, thus, are likely foreground or background stars rather than YSO cluster members. The remaining sources were fit with YSO models, and 27 of these met the $\chi^{2} / N_{\text {data }} \leq 4.8$ criterion. Of the nine 


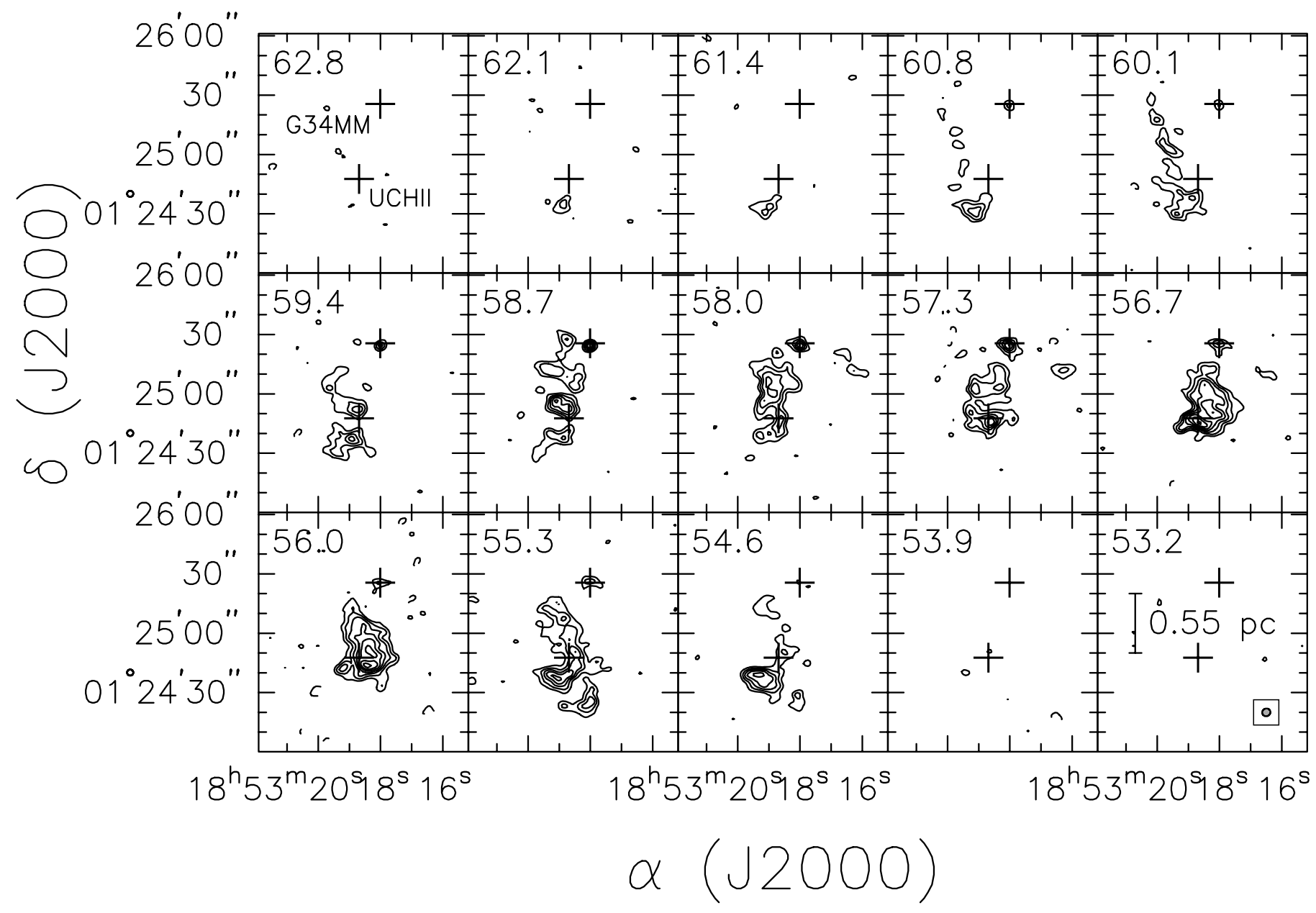

FIG. 7. $-\mathrm{C}^{18} \mathrm{O}(J=1-0)$ channel maps at $0.7 \mathrm{~km} \mathrm{~s}^{-1}$ spectral resolution. The central velocity is indicated in the top left corner of each panel. The channel rms is $60 \mathrm{mJy}$ beam ${ }^{-1}$, the peak is $0.92 \mathrm{Jy}_{\text {beam }}^{-1}$, and contours are plotted at $\pm 3,5,7,9,11,13$, and $15 \sigma$. The bottom right panel shows the synthesized beam in the bottom right corner $\left(4.04^{\prime \prime} \times 3.83^{\prime \prime}\right.$ at P.A. $\left.=-50.1^{\circ}\right)$ and a scale size of 0.55 pc. Plus symbols represent the locations of the UC H II region at the $6 \mathrm{~cm}$ continuum peak and G34.4 MM as indicated in the top left panel.

sources that were not well fit either by stellar atmospheres or YSO SEDs, one appears to be a YSO and eight are probably archive sources with improperly extracted fluxes in one or more bands or there was a mismatch of GLIMPSE with 2MASS or MIPS sources.

An overview of the results of the SED-fitting procedure is presented in Figure 10 and Tables 3 and 4. Most of the filamentary dark cloud is visible in the three-color image (Fig. 10) in which red corresponds to MIPS $24 \mu \mathrm{m}$, green to GLIMPSE $8 \mu \mathrm{m}$, and blue to GLIMPSE $4.5 \mu \mathrm{m}$. The G34.4 complex is located near the center of Figure 10 (in the region outlined with a large, white circle). Point sources are marked by small circles color coded by source type as identified by the Fit flag in Table 3. Sources that were well fit as YSOs are marked by green circles and given a Fit flag of 1 in Table 3. The yellow circle identifies a probable YSO that was not well fit $($ Fit $=2)$. Blue circles identify sources that were well fit by stellar photospheres. MIPS $24 \mu \mathrm{m}$ point sources that are detected in fewer than three of the 2MASS+IRAC bands and, hence, were not run through the fitter $($ Fit $=4)$ are identified by red circles; the majority of these are probably YSOs. The cyan circle (source 32 located between sources 8 and 10 in Fig. 10) marks the archive source that coincides with the proposed origin of outflow G34.4:D; it is detected only at 3.6 and $4.5 \mu \mathrm{m}$ and, hence, was not run through the fitter $($ Fit $=3)$. The area covered by the OVRO observations is contained primarily within the large white circle, which is referred to as region $\mathrm{C}$ in Table 3 . Region $\mathrm{N}$ is identified by the box north of region $\mathrm{C}$, while region $\mathrm{S}$ is identified by the box to the south. The G34.4 UC H II region appears white in this image because it is bright in diffuse emission at all wavelengths. There are two prominent MIPS sources: G34.4 MM and another, fainter source located about $2^{\prime}$ north of G34.4 MM at position $18^{\mathrm{h}} 53^{\mathrm{m}} 20.602^{\mathrm{s}},+01^{\circ} 28^{\prime} 25.61^{\prime \prime}$.

Table 3 presents a summary of the positions and fluxes in each band for all identified probable YSO cluster members in and near the dark cloud. Table 4 presents the best-fit model parameters for each YSO with enough flux points to be fitted. Output model parameters are: interstellar extinction to the source, $A_{V}$; stellar mass, $M_{\star}$; total luminosity of the YSO, $L_{\star}$; envelope accretion rate, $\dot{M}_{\text {env }}$; and inclination, $i$. Note that $A_{V}$ does not include the extinction produced by a circumstellar disk or envelope, and thus, it represents a lower limit for YSOs with significant circumstellar material. In general, each source can be well fit by multiple YSO SED models, and this generates a corresponding range of the best-fit model parameters. For well-fit YSOs, results of all fits with $\Delta \chi^{2} / N_{\text {data }} \leq 1$ relative to the best-fit model are reported. For example, for a typical source detected in $N_{\text {data }}=5$ bands, all fits with $\Delta \chi^{2} \geq 5$ with a formal probability relative to the best fit of less than $\exp \left(-\Delta \chi^{2} / 2\right)=0.08$ are excluded. The cumulative probability distribution for each parameter is then constructed using the Gaussian-weighted probability of each fit relative to the best fit. A range of parameter values that has a greater than 

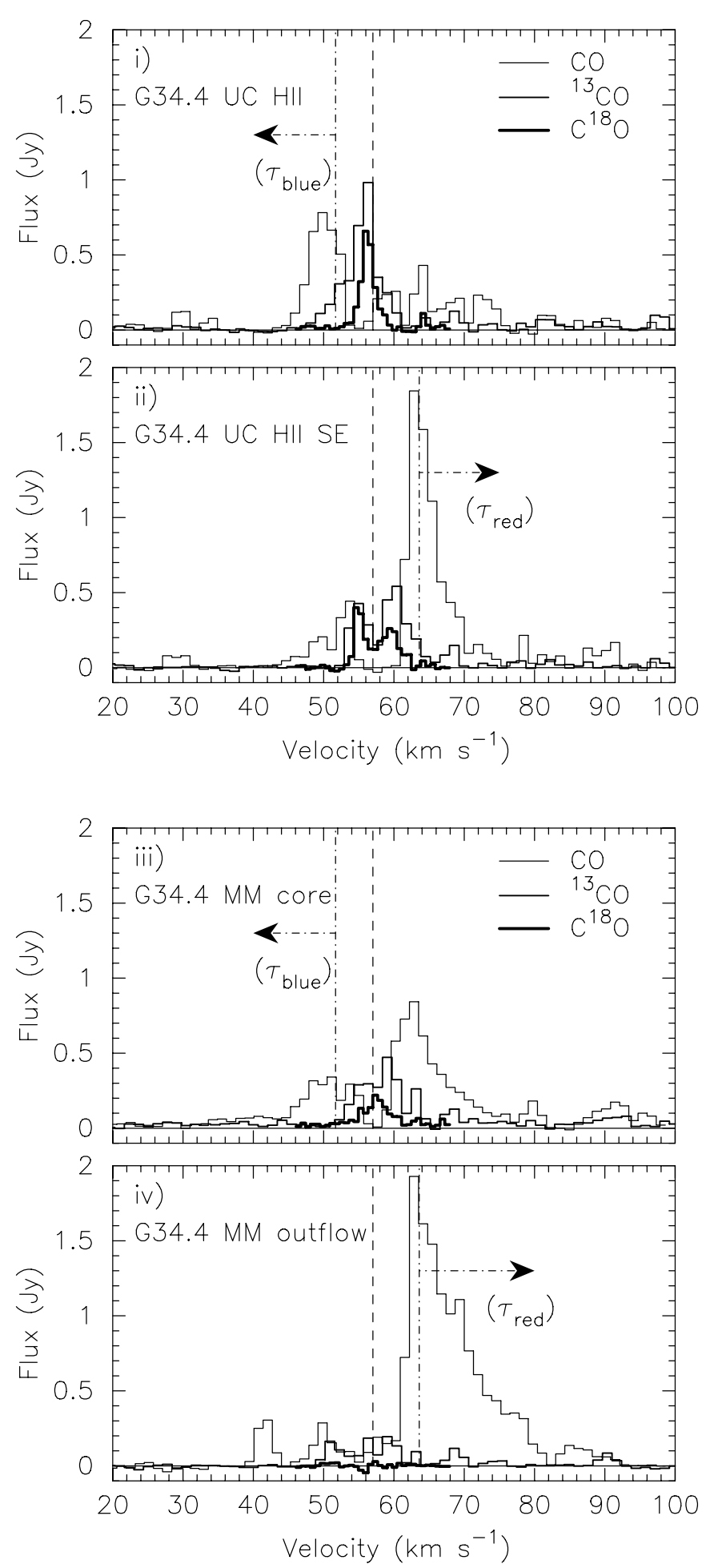

FIG. 8. - Spectra convolved with a $10^{\prime \prime}$ beam taken in $\mathrm{CO},{ }^{13} \mathrm{CO}$, and $\mathrm{C}^{18} \mathrm{O}$ at different locations in the image. The ratio of the $\mathrm{CO}$ and ${ }^{13} \mathrm{CO}$ emission was used to calculate optical depth in the red- and blueshifted outflow lobes as indicated by the dash-dotted lines and arrows in the figure. Panels ( $i$ ) and (ii) show spectra taken near the G34.4 UC H II region at positions $18^{\mathrm{h}} 53^{\mathrm{m}} 18.320^{\mathrm{s}}, 1^{\circ} 24^{\prime} 45.84^{\prime \prime}$ and $18^{\mathrm{h}} 53^{\mathrm{m}} 19.203^{\mathrm{s}}, 1^{\circ} 24^{\prime} 39.38^{\prime \prime}$, respectively. Panels (iii) and (iv) show spectra taken near the G34 MM core at positions $18^{\mathrm{h}} 53^{\mathrm{m}} 18.065^{\mathrm{s}}, 1^{\circ} 25^{\prime} 25.06^{\prime \prime}$ and $18^{\mathrm{h}} 53^{\mathrm{m}} 17.284^{\mathrm{s}}, 1^{\circ} 25^{\prime} 13.52^{\prime \prime}$, respectively. The locations of the spectra relative to the ${ }^{13} \mathrm{CO}$ emission are shown in Fig. 4 (top middle). The dashed vertical line at $57 \mathrm{~km} \mathrm{~s}^{-1}$ helps to illustrate that the peak of the $\mathrm{C}^{18} \mathrm{O}$ line shifts from $57.4 \mathrm{~km} \mathrm{~s}^{-1}$ toward the G34 MM core to $56 \mathrm{~km} \mathrm{~s}^{-1}$ toward the G34.4 UC H II region core.
$95 \%$ probability of containing the actual value for the source is then determined and reported in Table 4.

\subsubsection{The Central Region of the Filament}

In the region for which $\mathrm{CO}$ outflow data is determined, seven YSOs with good fits have been identified (sources 6-12), along with one poorly fit, possibly massive YSO (source 31 in Tables 3 and 4) located on the northeast edge of the G34.4 MM $24 \mu \mathrm{m}$ emission. Source 12, the highly luminous, the mid-IR counterpart to G34.4 MM, is most likely the driving source of outflow G34.4:A. Marked by a larger green circle in Figure 10, source 12 is partially resolved in the GLIMPSE images (this is seen most clearly in the $4.5 \mu \mathrm{m}$ gray scale image in Fig. 9). The morphology of source $12 / \mathrm{G} 34.4 \mathrm{MM}$ is consistent with two dust emission lobes separated by an absorption lane due to a disk where the lobes are in rough alignment with the axis of outflow G34.4:A.

The fluxes in the two lobes of source 12/G34.4 MM in each GLIMPSE band was summed and combined with the point-source fluxes from the Midcourse Space Experiment (MSX) $21.3 \mu \mathrm{m}$ (Price et al. 2001), MIPS $24 \mu \mathrm{m}$, and MIPS $70 \mu \mathrm{m}$ to model the source SED across the mid-IR. The 10 best fits to source 12/G34.4 MM are plotted in Figure 11. The fit parameters are well constrained and the best fit has $\chi^{2}=6.62$, which is significantly lower than the second-best fit with $\chi^{2}=20.79$. The bestfit parameters suggest a massive YSO with $M_{\star} \sim 14 M_{\odot}$ and $L_{\star} \sim 9400 L_{\odot}$. The source is deeply embedded behind greater than $A_{V} \sim 40$ mag of extinction, and it is young with a high envelope accretion rate of $\dot{M}_{\text {env }} \sim 10^{-3} M_{\odot} \mathrm{yr}^{-1}$.

The inclination angle of the best-fit model is $i=56^{\circ}$, consistent with the orientation of outflow G34.4:A, which has a wide opening angle of $43^{\circ}$ but does not intersect the plane of the sky. This interpretation assumes that a single YSO dominates the flux density and geometry of the mid-IR emission. Given that two outflows have been identified emanating from the G34.4 MM core and the far-IR fluxes exceed those predicted by a single YSO SED, more than one YSO is likely present. However, the resolution of the GLIMPSE images is not adequate to clearly distinguish between sources that are likely to drive outflows G34.4:A and G34.4:B.

For the remaining six well-fit YSOs in region $\mathrm{C}$, the best-fit models are presented in Figure 12a, while the distribution of their corresponding best-fit masses and luminosities are shown in Figure $12 b$. Of these, two YSOs (sources 10 and 11) are nearly coincident with the $\mathrm{UC} \mathrm{H}$ II region.

Source 11 is a luminous YSO located near the projected center of the UC H II region and appears to be the most likely candidate to drive outflow G34.4:C. The GLIMPSE position differs from the $6 \mathrm{~cm}$ ionized gas peak by $\sim 1.3^{\prime \prime}$. This could be due to differences in astrometry between the radio and IR data; however, the discrepancy exceeds the GLIMPSE astrometric uncertainty of $0.3^{\prime \prime}$ and the Very Large Array estimated uncertainty of $\sim 0.1^{\prime \prime}$. It is more likely that the centimeter peak tracing the ionized gas is offset from the actual YSO. This could easily happen if the ionized gas morphology closely follows the confines of a bipolar outflow cavity. It is also possible that the center of the source is not detected in the IRAC bands due to high extinction or the bright diffuse background of the $\mathrm{UC} \mathrm{H}$ II region itself. We may only see the warm outflow cavity associated with the blueshifted lobe in the mid-IR that is displaced from the centimeter continuum emission.

Both sources 10 and 11 suffer from confusion with the UC H II region at IR wavelengths longward of the IRAC bands. The UC $\mathrm{H}$ II region emits very strongly in all four $M S X$ bands and in MIPS 24 and $70 \mu \mathrm{m}$. These fluxes were used as upper limits for 
TABLE 1

G34.4 and G34 MM Outflow Parameters

\begin{tabular}{|c|c|c|}
\hline Parameter & G34.4 Combined Flows & G34 MM Combined Flows \\
\hline 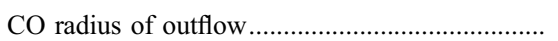 & $0.98 \mathrm{pc}$ & $0.85 \mathrm{pc}$ \\
\hline 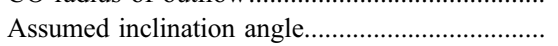 & $45^{\circ}$ & $45^{\circ}$ \\
\hline Redshifted outflow mass: ${ }^{12} \mathrm{CO}^{\mathrm{a}}$. & $22.6 M_{\odot}$ & $10.6 M_{\odot}$ \\
\hline Redshifted outflow mass: ${ }^{13} \mathrm{CO}^{\mathrm{a}}$. & $59.6 M_{\odot}$ & $12.7 M_{\odot}$ \\
\hline 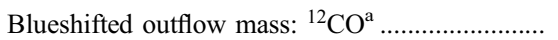 & $14.2 M_{\odot}$ & $1.6 M_{\odot}$ \\
\hline 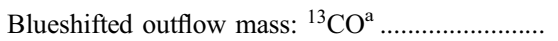 & $14.8 M_{\odot}$ & $9.9 M_{\odot}$ \\
\hline 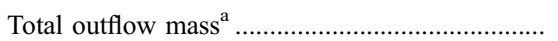 & $111.2 M_{\odot}$ & $34.8 M_{\odot}$ \\
\hline Momentum & $8.3 \times 10^{2} M_{\odot} \mathrm{km} \mathrm{s}^{-1}$ & $3.5 \times 10^{2} M_{\odot} \mathrm{km} \mathrm{s}^{-1}$ \\
\hline 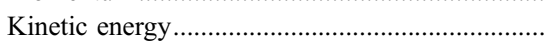 & $7.8 \times 10^{46} \mathrm{ergs}$ & $4.9 \times 10^{46} \mathrm{ergs}$ \\
\hline 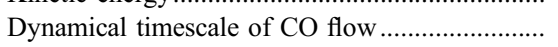 & $\sim 4 \times 10^{4} \mathrm{yr}$ & $\sim 3.8 \times 10^{4} \mathrm{yr}$ \\
\hline$\dot{M}_{f}$ & $1.3 \times 10^{-3} M_{\odot} \mathrm{yr}^{-1}$ & $0.8 \times 10^{-3} M_{\odot} \mathrm{yr}^{-1}$ \\
\hline 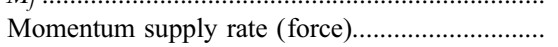 & $12.2 \times 10^{-3} M_{\odot} \mathrm{km} \mathrm{s}^{-1} \mathrm{yr}^{-1}$ & $8.0 \times 10^{-3} M_{\odot} \mathrm{km} \mathrm{s}^{-1} \mathrm{yr}^{-1}$ \\
\hline 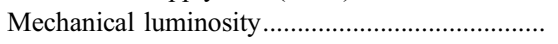 & $11.2 L_{\odot}$ & $9.1 L_{\odot}$ \\
\hline
\end{tabular}

the fits to sources 10 and 11 , but they are very high limits (see Fig. 12a), and the fits to both sources are poorly constrained. The best-fit results for source 11 suggest $M_{\star}=2-10 M_{\odot}$ and $L_{\star}=$ 200-5200 $L_{\odot}$, but the probability distribution of fit parameters shown in Figure $12 b$ is skewed and allows for higher masses and luminosities up to $M_{\star} \sim 20 M_{\odot}$ and $L_{\star} \sim 45,000 L_{\odot}$ (albeit at a formal probability level of only a few percent). SNB04 determined a spectral type of B0.5 $\left(L_{\star} \sim 40,000 L_{\odot}\right)$ for the central star of the G34.4 UC H II region assuming a single zero-age main-sequence star is producing the observed Lyman continuum flux. Thus, if source 10 is the ionizing star of the $\mathrm{UC} \mathrm{H}$ in region, the higher values of mass and luminosity are more plausible.

Due to its physical location between the red- and blueshifted lobes of outflow G34.4:E, source 10 appears to be the most probable driver of this outflow. The best-fit results suggest that it is a YSO with $M_{\star}=0.5-10 M_{\odot}$ and $L_{\star}=45-4500 L_{\odot}$. Again, the association of this YSO with strong outflow activity suggests that the upper end of this range of mass and luminosity may be more appropriate.

Source 32 (cyan circle), the potential driver of outflow G34.4:D, is detected in only two IRAC bands ([3.6] and [4.5]) at the $5 \sigma$ level, sufficient for inclusion into the GLIMPSE archive. Based on these two bands, source 32 has a red color of [3.6] - [4.5] $=1.3$ which is consistent with it being a YSO (Robitaille et al. 2006). The lack of GLIMPSE archive fluxes in bands [5.8] and [8.0] means that this source was not detected at the $3 \sigma$ level in those two bands, corresponding to magnitudes fainter than 13.0 in each band. ${ }^{11}$ The colors of YSO models for very young sources pre$\operatorname{dict}[3.6]-[5.8]>0.4$ and $[5.8]-[8.0]>0.4$, corresponding to magnitudes fainter than 13.6 and 13.2 in bands [5.8] and [8.0],

${ }^{11}$ See the GLIMPSE Quality Assurance document at http://www.astro.wisc .edu/glimpse/GQA-master.pdf.

TABLE 2

G34.4 and G34 MM Core Parameters Measured from $\mathrm{C}^{18} \mathrm{O}$

\begin{tabular}{|c|c|c|}
\hline Parameter & G34.4 & G34 MM \\
\hline 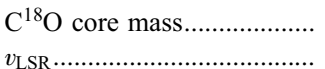 & $\begin{array}{l}692.7 M_{\odot} \\
56.0 \mathrm{~km} \mathrm{~s}^{-1}\end{array}$ & $\begin{array}{c}75.2 M_{\odot} \\
57.4 \mathrm{~km} \mathrm{~s}^{-1}\end{array}$ \\
\hline Momentum ................................ & $2.0 \times 10^{3} M_{\odot} \mathrm{km} \mathrm{s}^{-1}$ & $2.5 \times 10^{2} M_{\odot} \mathrm{km} \mathrm{s}^{-1}$ \\
\hline Kinetic energy ......................... & $8.0 \times 10^{46} \mathrm{ergs}$ & $1.2 \times 10^{46} \mathrm{ergs}$ \\
\hline
\end{tabular}

respectively (Robitaille et al. 2006). Thus, the nondetection in bands [5.8] and [8.0] is consistent with the interpretation that source 32 is a YSO.

Source 32 is also not detected in the MIPS $24 \mu \mathrm{m}$ band. However, given the proximity to the very bright UC H II and lower resolution of MIPS compared to IRAC, this source is confused with the point-spread function wings of the UC $\mathrm{H}$ II region. Based on an estimate of the MIPS sensitivity in a confused region, a $3 \sigma$

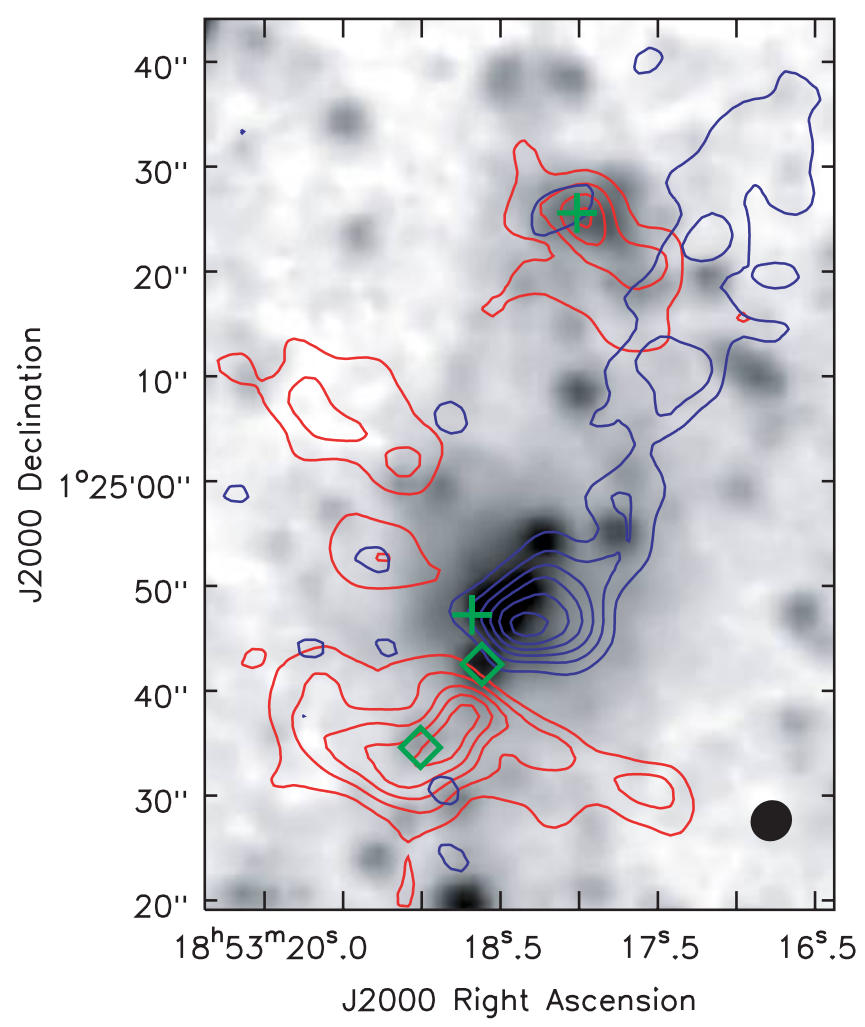

FIG. 9.-GLIMPSE $4.5 \mu \mathrm{m}$ image in inverted gray scale showing locations of G34.4 MM and G34.4 UC H II region as plus signs. Diamonds show the predicted locations of the driving sources of outflows G34.4:D and G34.4:E based on CO and ${ }^{13} \mathrm{CO}$ morphology. GLIMPSE sources are located very near the predicted positions, suggesting that GLIMPSE has detected two sources driving outflows D and E. ${ }^{13} \mathrm{CO}$ red- and blueshifted emission contours from Fig. 4 are overlaid for reference. 


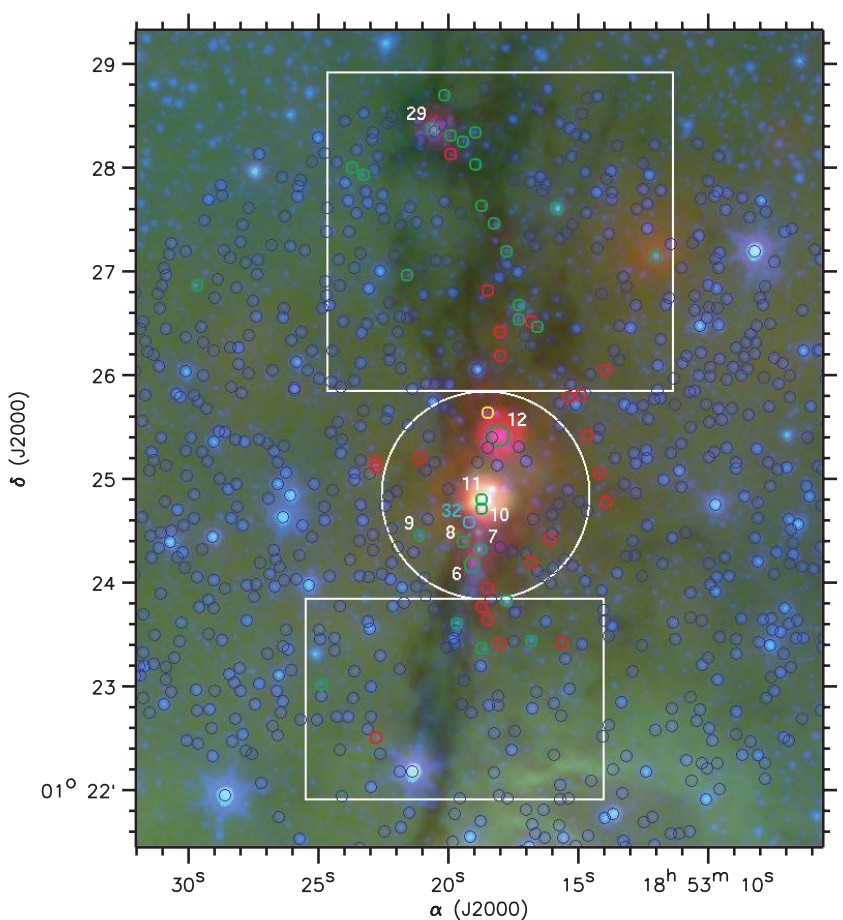

FIG. 10.-GLIMPSE and MIPSGAL three-color image with 4.5 (blue), 8.0 ( green), and $24 \mu \mathrm{m}$ (red). The probable YSO cluster members listed in Table 3 are marked by color-coded circles: green are good YSO fits $\left(\chi^{2} / N_{\text {data }} \leq 4.8\right)$, yellow are bad YSO fits $\left(\chi^{2} / N_{\text {data }}>14.8\right)$, cyan are sources detected in fewer than four bands, and red are MIPS $24 \mu \mathrm{m}$ point sources detected in too few IRAC +2 MASS bands to be fit. Sources well fit by stellar photospheres are marked by blue circles. The IR-dark cloud has been divided into three regions for the purpose of the YSO census: region $\mathrm{C}$ (large white circle) contains the OVRO observations, and regions $\mathrm{N}$ and $\mathrm{S}$ (large white rectangles) are the portions of the dark cloud lying to the north and south, respectively, of region C. Source numbers from Table 3 for the YSOs discussed in $\S 3.4$ are also shown.

upper limit of $50 \mathrm{mJy}$ (magnitude 5.4) is expected for the $24 \mu \mathrm{m}$ flux. This could potentially allow a very red $[3.6]-[24] \approx 8.6$ color for this source. We therefore conclude that the mid-IR colors of source 32 are consistent with those for a YSO; however, the strongest evidence for this remains the fact that it lies along the $\mathrm{CO}$ outflow axis of G34.4:D near where one might expect the driving source to be located.

In summary, nine YSOs have been identified and characterized in region $\mathrm{C}$ near G34.4 MM and the $\mathrm{UC} H$ II region. Four or five are probably massive with $M_{\star}>8 M_{\odot}$, while the others have low to intermediate mass with $M_{\star}$ between 0.5 and $8 M_{\odot}$.

\subsubsection{The Northern Filament}

North of the G34.4 MM and UC H II region complex (in region N), 17 YSOs have been successfully identified based on fits to the SEDs. Most of these YSOs closely trace the IR-dark cloud structure. At the extreme north end of the field, there is another $24 \mu \mathrm{m}$ bright source (source 29 in Tables 3 and 4) that may be similar to but less massive than G34.4 MM.

The SED is well fit with $\chi^{2}=17.39$, suggesting a YSO with $M_{\star} \sim 4.8-6.8 M_{\odot}$ and $L_{\star} \sim 170-340 L_{\odot}$. The remaining 16 YSOs in region $\mathrm{N}$ are less massive than source 29 with mass estimates between about 0.5 and $6.5 M_{\odot}$.

\subsubsection{The Southern Filament}

The southern part of the IR-dark filament (region S) contains five sources with SEDs that are well fit as YSOs. Five relatively faint $24 \mu \mathrm{m}$ sources that are likely to be YSOs are also scattered throughout this region. Sources in this southern region are more distributed and are not spatially well correlated with the dark cloud filament. This is in contrast to the YSOs in the northern region where they follow the cloud boundaries reasonably well. Mass estimates range from about 1 to $7 M_{\odot}$.

\section{DISCUSSION}

The IR-dark cloud associated with IRAS $18507+0121$ has a filamentary structure that spans more than $9^{\prime}$ on the sky $(>10 \mathrm{pc}$ at $3.9 \mathrm{kpc}$ ). Splitting the cloud into three regions (south, central, and north), one finds that the southern region is associated with mostly low- to intermediate-mass YSO candidates (10 in total) that are not spatially well correlated with the dark cloud filament. The northern segment of the filament contains 23 low- to intermediatemass YSO candidates that tend to trace the IR-dark cloud structure. The central region of the filament is in the process of forming four or five mid to early B stars with $M_{\star}>8 M_{\odot}$. Four more sources are likely low to intermediate mass with $M_{\star}$ between 0.5 and $8 M_{\odot}$. Together with an additional 11 YSO candidates seen at $24 \mu \mathrm{m}$, this central region harbors a relatively compact cluster with a total of 20 YSO candidates.

\subsection{GLIMPSE-detected Source Characteristics}

Bright emission in the $4.5 \mu \mathrm{m}$ Spitzer IRAC can be a strong indicator of molecular outflow (e.g., Qiu et al. 2007; Smith et al. 2006; Noriega-Crespo et al. 2004). The most likely reason for this is the presence of $\mathrm{H}_{2}$ emission lines and/or emission from the $\mathrm{CO}(J=1-0) P(8)$ band head. All four IRAC bands contain $\mathrm{H}_{2}$ emission lines, but these features are narrow and, hence, can only significantly enhance the brightness in the broadband images if the lines are extremely bright relative to the continuum. Because $\mathrm{H}_{2}$ features are distributed throughout the IRAC bands, strong $\mathrm{H}_{2}$ lines will not cause a greater enhancement of the flux in band [4.5] than in bands [5.8] and [8.0] (see, e.g., Smith et al. 2006). In contrast, the $\operatorname{CO}(J=1-0) P(8)$ emission band head is a broad feature that occupies the entire [4.5] filter and can become bright in the presence of shocked, molecular gas. There is not significant $\mathrm{CO}$ band head emission in the remaining IRAC bands. Thus, sources with excess emission in band [4.5] relative to bands [5.8] and [8.0] appear likely to have $\mathrm{CO}$ band head emission resulting from shocked, molecular gas. This does not preclude the possibility that significant $\mathrm{H}_{2}$ emission may also contribute to the brightness in all IRAC bands.

Three of the 29 YSOs listed in Table 3 that were detected in all four IRAC bands have noticeable excess emission at $4.5 \mu \mathrm{m}$ and strong flux at $24 \mu \mathrm{m}$ : sources 6 and 12 in region $C$ and source 29 in region N. Because of this, SED fits used the [4.5] fluxes as upper limits only, since the YSO SED models do not incorporate molecular line emission. These sources are likely to have significant shocked, molecular gas and are undergoing outflow and accretion. Source 31, although it was not well fit, should probably be considered in this group as well. It was not well fit because it has a strong $4.5 \mu \mathrm{m}$ excess and it was only detected in the four IRAC bands. The SED (not shown) looks similar to G34.4 MM itself. Furthermore, its nondetection at $24 \mu \mathrm{m}$ is almost certainly due to its proximity to $\mathrm{G} 34.4 \mathrm{MM}$.

The most prominent of the $4.5 \mu \mathrm{m}$ excess sources is source 12 , the mid-IR counterpart to G34.4 MM and outflow G34.4:A. The association of this source with the $\mathrm{CO}$ outflow favors the interpretation that the $4.5 \mu \mathrm{m}$ excess is due to shocked $\mathrm{CO}$ emission.

The potential drivers of outflows G34.4:C (source 11) and G34.4:E (source 10) do not show similar $4.5 \mu$ m excess fluxes. A possible reason for this may be that they are somewhat more 
TABLE 3

YSO Cluster Members

\begin{tabular}{|c|c|c|c|c|c|c|c|c|c|c|c|c|c|c|}
\hline \multirow[b]{2}{*}{ SoURCE $^{\mathrm{a}}$} & \multicolumn{2}{|c|}{ CoOrdinates } & \multirow[b]{2}{*}{$\begin{array}{l}F[3.6] \\
(\mathrm{mJy})\end{array}$} & \multirow[b]{2}{*}{$\begin{array}{c}\delta F[3.6] \\
(\mathrm{mJy})\end{array}$} & \multicolumn{7}{|c|}{ MEAsured Mid-IR FluXes and UnCertainties } & \multirow[b]{2}{*}{$\begin{array}{c}\delta F[24]^{\mathrm{b}} \\
(\mathrm{mJy})\end{array}$} & \multirow[b]{2}{*}{ FIT $^{c}$} & \multirow[b]{2}{*}{ REGION $^{\mathrm{d}}$} \\
\hline & $\alpha(\mathrm{J} 2000.0)$ & $\delta(\mathrm{J} 2000.0)$ & & & $\begin{array}{l}F[4.5] \\
\text { (mJy) }\end{array}$ & $\begin{array}{c}\delta F[4.5] \\
(\mathrm{mJy})\end{array}$ & $\begin{array}{c}F[5.8] \\
(\mathrm{mJy})\end{array}$ & $\begin{array}{c}\delta F[5.8] \\
(\mathrm{mJy})\end{array}$ & $\begin{array}{c}F[8.0] \\
(\mathrm{mJy})\end{array}$ & $\begin{array}{c}\delta F[8.0] \\
(\mathrm{mJy})\end{array}$ & $\begin{array}{l}F[24] \\
\text { (mJy) }\end{array}$ & & & \\
\hline ........... & 185324.999 & 12301.4 & 3.43 & 0.34 & 2.11 & 0.23 & 1.86 & 0.37 & $\ldots$ & $\ldots$ & 1.61 & 0.16 & 1 & $\mathrm{~S}$ \\
\hline $2 \ldots \ldots \ldots \ldots \ldots$ & 185318.649 & 12321.8 & 1.78 & 0.18 & 2.01 & 0.20 & 2.85 & 0.28 & 2.68 & 0.40 & 10.54 & 1.68 & 1 & $\mathrm{~S}$ \\
\hline 3................ & 185316.833 & 12326.6 & 5.20 & 0.52 & 6.10 & 0.61 & 5.07 & 0.51 & 4.33 & 0.65 & 6.73 & 0.67 & 1 & $\mathrm{~S}$ \\
\hline 4................ & 185319.694 & 12336.2 & 6.78 & 0.68 & 16.79 & 1.68 & 28.13 & 2.81 & 20.32 & 3.05 & 60.00 & U.L. & 1 & $\mathrm{~S}$ \\
\hline $5 \ldots \ldots \ldots \ldots \ldots$ & 185317.773 & 12349.4 & 8.66 & 0.87 & 12.26 & 1.23 & 13.92 & 1.39 & 15.76 & 2.36 & 45.29 & 4.53 & 1 & $\mathrm{~S}$ \\
\hline 6.................... & 185318.982 & 12411.5 & 0.84 & 0.15 & 7.66 & 0.93 & 5.00 & 0.86 & 2.79 & 0.64 & 307.85 & 30.78 & 1 & $\mathrm{C}$ \\
\hline 7................... & 185318.825 & 12419.6 & 12.22 & 1.22 & 26.14 & 2.61 & 26.69 & 2.67 & 23.15 & 3.47 & 150.00 & U.L. & 1 & $\mathrm{C}$ \\
\hline $8 \ldots \ldots \ldots \ldots \ldots$ & 185319.404 & 12424.2 & 0.25 & 0.07 & 1.46 & 0.16 & 3.33 & 0.36 & 4.18 & 0.63 & 150.00 & U.L. & 1 & $\mathrm{C}$ \\
\hline$\ldots \ldots$. & 185321.127 & 12427.5 & 3.79 & 0.38 & 3.56 & 0.36 & 3.07 & 0.52 & 2.00 & 0.30 & 19.92 & 2.54 & 1 & $\mathrm{C}$ \\
\hline $10^{\mathrm{e}}$. & 185318.727 & 12443.0 & 5.13 & 0.92 & 19.57 & 1.96 & 40.99 & 6.81 & 59.72 & 8.96 & 18024.00 & U.L. & 1 & $\mathrm{C}$ \\
\hline $11^{\mathrm{e}} \ldots$ & 185318.630 & 12448.3 & 28.26 & 7.77 & 79.15 & 14.59 & 168.90 & 16.89 & 277.30 & 45.02 & 18024.00 & U.L. & 1 & $\mathrm{C}$ \\
\hline $12^{\mathrm{f}} \ldots \ldots \ldots \ldots \ldots$ & 185318.058 & 12525.3 & 2.24 & 0.22 & 16.20 & 8.00 & 13.85 & 1.38 & 11.34 & 1.13 & 8682.80 & 868.28 & 1 & $\mathrm{C}$ \\
\hline $13 \ldots \ldots \ldots \ldots \ldots$ & 185316.533 & 12628.6 & 0.37 & 0.06 & 1.03 & 0.10 & 1.40 & 0.22 & 1.59 & 0.24 & 7.11 & 0.71 & 1 & $\mathrm{~N}$ \\
\hline $14 \ldots \ldots \ldots \ldots \ldots$ & 185317.321 & 12633.0 & 1.88 & 0.19 & 2.65 & 0.27 & 2.83 & 0.40 & 1.77 & 0.27 & 15.01 & 1.50 & 1 & $\mathrm{~N}$ \\
\hline $15 \ldots \ldots \ldots \ldots \ldots$ & 185317.232 & 12641.1 & 5.01 & 0.50 & 6.90 & 0.69 & 7.18 & 0.72 & 6.23 & 0.93 & 11.21 & 1.12 & 1 & $\mathrm{~N}$ \\
\hline $16 \ldots \ldots \ldots \ldots$ & 185329.815 & 12652.7 & 3.30 & 0.33 & 4.12 & 0.41 & 4.68 & 0.53 & 5.80 & 0.87 & 8.09 & 0.81 & 1 & $\ldots$ \\
\hline $17 \ldots \ldots$ & 185321.571 & 12658.9 & 0.38 & 0.06 & 1.58 & 0.23 & 1.58 & 0.35 & $\ldots$ & $\ldots$ & 13.46 & 1.61 & 1 & $\mathrm{~N}$ \\
\hline $18 \ldots$ & 185311.979 & 12709.5 & 13.41 & 1.34 & 10.45 & 1.04 & 8.31 & 0.83 & 7.87 & 1.61 & 119.71 & 37.16 & 1 & $\mathrm{~N}$ \\
\hline $19 \ldots \ldots \ldots \ldots \ldots$ & 185317.787 & 12712.6 & 0.81 & 0.09 & 2.11 & 0.21 & 3.29 & 0.33 & 3.94 & 0.59 & 13.88 & 1.39 & 1 & $\mathrm{~N}$ \\
\hline $20 \ldots \ldots \ldots \ldots \ldots$ & 185318.181 & 12728.5 & 1.40 & 0.19 & 3.23 & 0.32 & 3.30 & 0.41 & 3.40 & 0.51 & 13.21 & 1.32 & 1 & $\mathrm{~N}$ \\
\hline $21 \ldots \ldots \ldots \ldots$ & 185315.807 & 12737.4 & 40.59 & 4.06 & 41.08 & 4.11 & 46.97 & 4.70 & 36.85 & 5.53 & 20.79 & 2.08 & 1 & $\mathrm{~N}$ \\
\hline $22 \ldots \ldots \ldots \ldots \ldots$ & 185318.604 & 12738.8 & 0.49 & 0.09 & 0.71 & 0.11 & $\ldots$ & $\ldots$ & 2.66 & 0.40 & 10.97 & 1.40 & 1 & $\mathrm{~N}$ \\
\hline $23 \ldots \ldots \ldots \ldots$ & 185323.391 & 12757.0 & 2.47 & 0.25 & 2.52 & 0.25 & 2.70 & 0.34 & 2.35 & 0.35 & 4.07 & 0.41 & 1 & $\mathrm{~N}$ \\
\hline $24 \ldots \ldots \ldots \ldots \ldots$ & 185323.784 & 12801.1 & 0.49 & 0.08 & 0.55 & 0.06 & 1.02 & 0.32 & 0.96 & 0.14 & 4.94 & 0.49 & 1 & $\mathrm{~N}$ \\
\hline $25 \ldots \ldots \ldots \ldots \ldots$ & 185318.848 & 12802.7 & 0.65 & 0.09 & 0.58 & 0.09 & $\ldots$ & $\ldots$ & 2.59 & 0.39 & $\ldots$ & $\ldots$ & 1 & $\mathrm{~N}$ \\
\hline $26 \ldots \ldots \ldots \ldots \ldots$ & 185319.404 & 12816.1 & 0.83 & 0.09 & 2.12 & 0.21 & 2.60 & 0.42 & 2.93 & 0.44 & 4.16 & 0.42 & 1 & $\mathrm{~N}$ \\
\hline $27 \ldots \ldots \ldots \ldots \ldots$ & 185320.005 & 12819.6 & 0.55 & 0.08 & 1.29 & 0.23 & 2.14 & 0.68 & 1.47 & 0.22 & 29.57 & 2.96 & 1 & $\mathrm{~N}$ \\
\hline $28 \ldots \ldots \ldots \ldots \ldots$ & 185319.049 & 12821.5 & 2.53 & 0.29 & 2.64 & 0.26 & 2.23 & 0.24 & 1.68 & 0.25 & 5.42 & 0.54 & 1 & $\mathrm{~N}$ \\
\hline $29 \ldots \ldots \ldots \ldots \ldots$ & 185320.674 & 12824.1 & 9.65 & 0.96 & 32.79 & 3.94 & 26.83 & 2.68 & 8.03 & 1.20 & 278.27 & 27.83 & 1 & $\mathrm{~N}$ \\
\hline $30 \ldots \ldots \ldots \ldots \ldots$ & 185320.078 & 12843.1 & 0.27 & 0.05 & 1.02 & 0.11 & 1.83 & 0.28 & 2.49 & 0.37 & 15.80 & 1.58 & 1 & $\mathrm{~N}$ \\
\hline 31............... & 185318.586 & 12538.9 & 0.33 & 0.08 & 3.43 & 0.39 & 2.50 & 0.32 & 1.79 & 0.27 & $\ldots$ & $\ldots$ & 2 & $\mathrm{C}$ \\
\hline $32 \ldots \ldots \ldots \ldots \ldots$ & 185319.144 & 12435.4 & 0.68 & 0.09 & 1.46 & 0.18 & $\ldots$ & $\ldots$ & $\ldots$ & $\ldots$ & $\ldots$ & $\ldots$ & 3 & $\mathrm{C}$ \\
\hline 33................. & 185313.870 & 12603.3 & 0.98 & 0.13 & 0.71 & 0.11 & $\ldots$ & $\ldots$ & $\ldots$ & $\ldots$ & 3.31 & 0.59 & 4 & $\mathrm{~N}$ \\
\hline $34 \ldots \ldots \ldots \ldots \ldots$ & 185313.933 & 12446.7 & $\ldots$ & $\ldots$ & $\ldots$ & $\ldots$ & $\ldots$ & $\ldots$ & $\ldots$ & $\ldots$ & 5.79 & 0.40 & 4 & $\ldots$ \\
\hline $35 \ldots \ldots \ldots \ldots \ldots$ & 185314.135 & 12503.8 & 0.35 & 0.06 & 0.34 & 0.06 & $\ldots$ & $\ldots$ & $\ldots$ & $\ldots$ & 5.52 & 1.00 & 4 & $\ldots$ \\
\hline $36 \ldots \ldots \ldots \ldots \ldots$ & 185314.702 & 12525.0 & $\ldots$ & $\ldots$ & $\ldots$ & $\ldots$ & $\ldots$ & $\ldots$ & $\ldots$ & $\ldots$ & 8.00 & 0.99 & 4 & $\ldots$ \\
\hline $37 \ldots \ldots \ldots \ldots$ & 185314.884 & 12549.0 & $\ldots$ & $\ldots$ & $\ldots$ & $\ldots$ & $\ldots$ & $\ldots$ & $\ldots$ & $\ldots$ & 4.56 & 0.50 & 4 & $\ldots$ \\
\hline $38 \ldots \ldots \ldots \ldots \ldots$ & 185315.298 & 12548.1 & 1.27 & 0.15 & 0.86 & 0.09 & $\ldots$ & $\ldots$ & $\ldots$ & $\ldots$ & 11.14 & 1.11 & 4 & $\ldots$ \\
\hline $39 \ldots \ldots \ldots \ldots$ & 185315.562 & 12325.2 & $\ldots$ & $\ldots$ & $\ldots$ & $\ldots$ & $\ldots$ & $\ldots$ & $\ldots$ & $\ldots$ & 2.75 & 0.53 & 4 & $\mathrm{~S}$ \\
\hline $40 \ldots \ldots \ldots \ldots \ldots$ & 185316.029 & 12425.4 & $\ldots$ & $\ldots$ & $\ldots$ & $\ldots$ & $\ldots$ & $\ldots$ & $\ldots$ & $\ldots$ & 21.63 & 1.27 & 4 & $\mathrm{C}$ \\
\hline $41 \ldots \ldots \ldots \ldots$ & 185316.811 & 12411.9 & $\ldots$ & $\ldots$ & $\ldots$ & $\ldots$ & $\ldots$ & $\ldots$ & $\ldots$ & $\ldots$ & 10.22 & 0.47 & 4 & $\mathrm{C}$ \\
\hline $42 \ldots \ldots \ldots \ldots \ldots$ & 185316.908 & 12632.0 & $\ldots$ & $\ldots$ & $\ldots$ & $\ldots$ & $\ldots$ & $\ldots$ & $\ldots$ & $\ldots$ & 8.87 & 0.92 & 4 & $\mathrm{~N}$ \\
\hline $43 \ldots \ldots \ldots \ldots \ldots$ & 185318.026 & 12625.9 & $\ldots$ & $\ldots$ & $\ldots$ & $\ldots$ & $\ldots$ & $\ldots$ & $\ldots$ & $\ldots$ & 42.84 & 2.55 & 4 & $\mathrm{~N}$ \\
\hline $44 \ldots \ldots \ldots \ldots \ldots$ & 185318.065 & 12324.8 & $\ldots$ & $\ldots$ & $\ldots$ & $\ldots$ & $\ldots$ & $\ldots$ & $\ldots$ & $\ldots$ & 7.76 & 0.97 & 4 & $\mathrm{~S}$ \\
\hline $45 \ldots \ldots \ldots \ldots \ldots$ & 185318.085 & 12612.0 & 0.49 & 0.07 & 0.45 & 0.08 & $\ldots$ & $\ldots$ & $\ldots$ & $\ldots$ & 8.27 & 3.97 & 4 & $\mathrm{~N}$ \\
\hline $46 \ldots \ldots \ldots \ldots$ & 185318.409 & 12338.7 & $\ldots$ & $\ldots$ & $\ldots$ & $\ldots$ & $\ldots$ & $\ldots$ & $\ldots$ & $\ldots$ & 10.10 & 0.83 & 4 & $\mathrm{~S}$ \\
\hline $47 \ldots \ldots \ldots \ldots \ldots$ & 185318.516 & 12356.8 & $\ldots$ & $\ldots$ & $\ldots$ & $\ldots$ & $\ldots$ & $\ldots$ & $\ldots$ & $\ldots$ & 17.53 & 4.67 & 4 & $\mathrm{C}$ \\
\hline $48 \ldots \ldots \ldots \ldots$ & 185318.589 & 12649.5 & $\ldots$ & $\ldots$ & $\ldots$ & $\ldots$ & $\ldots$ & $\ldots$ & $\ldots$ & $\ldots$ & 4.47 & 0.50 & 4 & $\mathrm{~N}$ \\
\hline $49 \ldots \ldots \ldots \ldots$ & 185318.600 & 12346.2 & $\ldots$ & $\ldots$ & $\ldots$ & $\ldots$ & $\ldots$ & $\ldots$ & $\ldots$ & $\ldots$ & 20.59 & 2.42 & 4 & $\mathrm{~S}$ \\
\hline $50 \ldots \ldots \ldots \ldots$ & 185319.904 & 12808.6 & $\ldots$ & $\ldots$ & 0.68 & 0.08 & 1.30 & 0.36 & $\ldots$ & $\ldots$ & 12.96 & 1.30 & 4 & $\mathrm{~N}$ \\
\hline $51 \ldots \ldots \ldots \ldots . .$. & 185321.233 & 12512.2 & $\ldots$ & $\ldots$ & $\ldots$ & $\ldots$ & $\ldots$ & $\ldots$ & $\ldots$ & $\ldots$ & 14.51 & 0.83 & 4 & $\mathrm{C}$ \\
\hline $52 \ldots \ldots \ldots \ldots$ & 185322.760 & 12230.3 & $\ldots$ & $\ldots$ & $\ldots$ & $\ldots$ & $\ldots$ & $\ldots$ & $\ldots$ & $\ldots$ & 6.44 & 0.95 & 4 & $\mathrm{~S}$ \\
\hline $53 \ldots \ldots \ldots \ldots$ & 185322.826 & 12510.0 & 0.80 & 0.10 & 0.67 & 0.10 & $\ldots$ & $\ldots$ & $\ldots$ & $\ldots$ & 35.49 & U.L. & 4 & $\ldots$ \\
\hline $54 \ldots \ldots \ldots \ldots \ldots$ & 185322.905 & 12507.0 & 1.58 & 0.16 & 1.53 & 0.18 & $\ldots$ & $\ldots$ & $\ldots$ & $\ldots$ & 35.49 & U.L. & 4 & $\ldots$ \\
\hline
\end{tabular}

NotE.- Units of right ascension are hours, minutes, and seconds, and units of declination are degrees, arcminutes, and arcseconds.

a Sources are grouped in order of Fit flag and then listed in order of increasing declination.

b Where two or more GLIMPSE sources are confused with a single MIPS source, we treat the $24 \mu \mathrm{m}$ flux as an upper limit (U.L.) only.

c The Fit flags are designated as follows: $1=$ Source well fit as a YSO, meeting the $\chi^{2} / N_{\text {data }} \leq 4.8$ criterion discussed in the text. $2=$ Fit failed to meet the $\chi^{2}$ criterion,

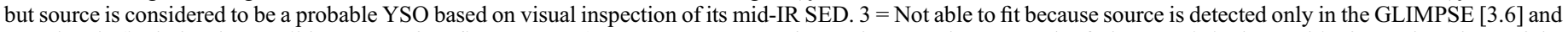

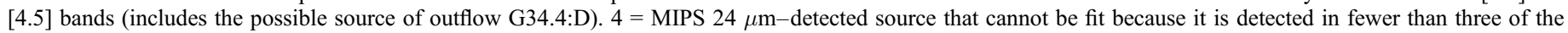
IRAC+2MASS bands. The majority of these sources are probably YSOs due to their location near the IR-dark cloud, but a few could be field stars on the asymptotic giant branch.

${ }^{\mathrm{d}}$ Region of Fig. 11 in which the source is located. Region C refers to the central part of the IR-dark cloud containing the OVRO observations. Region N represents the dark cloud north of $\mathrm{C}$, while region $\mathrm{S}$ represents the dark cloud south of $\mathrm{C}$. Unlabeled sources lie outside of these three regions.

e These sources overlie the UC H II region, which produces bright diffuse emission in all four MSX bands as well as at MIPS 24 and $70 \mu$ m. Although these YSOs may be bright enough to be detected by MSX and MIPS, they are confused with the UC H II region.

${ }^{\mathrm{f}}$ The IR counterpart to G34.4 MM. It is detected also by $M S X$ at $21.3 \mu \mathrm{m}$ and by MIPS at $70 \mu \mathrm{m}$, with fluxes of $4900 \pm 258$ and $322,000 \pm 128,000 \mathrm{mJy}$, respectively. 
TABLE 4

YSO Model Parameters ${ }^{\mathrm{a}}$ from SED Fits to Cluster Members

\begin{tabular}{|c|c|c|c|c|c|c|c|c|c|}
\hline Source & Fit & $\chi_{\min }^{2}$ & $\chi_{\max }^{2}$ & No. of Fits & $A_{V}$ & $\begin{array}{c}M_{\star} \\
\left(M_{\odot}\right) \\
\end{array}$ & $\begin{array}{c}L_{\star} \\
\left(L_{\odot}\right)\end{array}$ & $\begin{array}{c}\dot{M}_{\mathrm{env}} \\
\left(M_{\odot} \mathrm{yr}^{-1}\right)^{\mathrm{c}}\end{array}$ & $i$ \\
\hline $1 \ldots$ & 1 & 7.63 & 13.57 & 112 & $8.5-15.1$ & $2.63 \mathrm{E}+00-4.34 \mathrm{E}+00$ & $3.44 \mathrm{E}+01-2.83 \mathrm{E}+02$ & 0 & $18.2-87.1$ \\
\hline ....... & 1 & 8.24 & 14.21 & 96 & $11.4-22.8$ & $2.65 \mathrm{E}+00-4.24 \mathrm{E}+00$ & $4.82 \mathrm{E}+01-1.18 \mathrm{E}+02$ & $0-3.06 \mathrm{E}-07$ & $41.4-81.4$ \\
\hline ................... & 1 & 8.18 & 12.08 & 29 & $22.5-58.3$ & $1.03 \mathrm{E}+00-6.77 \mathrm{E}+00$ & $1.10 \mathrm{E}+02-1.54 \mathrm{E}+03$ & 0 & $18.2-81.4$ \\
\hline 5 & 1 & 0.86 & 7.85 & 185 & $11.4-26.5$ & $3.32 \mathrm{E}+00-5.03 \mathrm{E}+00$ & $1.03 \mathrm{E}+02-3.86 \mathrm{E}+02$ & $0-8.44 \mathrm{E}-06$ & $18.2-81.4$ \\
\hline 8 & 1 & 2.83 & 6.82 & 323 & $0.0-59.9$ & $1.94 \mathrm{E}-01-5.18 \mathrm{E}+00$ & $4.75 \mathrm{E}+00-2.27 \mathrm{E}+02$ & $0-4.22 \mathrm{E}-04$ & $18.2-75.5$ \\
\hline $9 \ldots .$. & 1 & 6.46 & 12.42 & 36 & $0.0-13.2$ & $9.22 \mathrm{E}-01-3.34 \mathrm{E}+00$ & $9.97 \mathrm{E}+00-3.53 \mathrm{E}+01$ & $1.18 \mathrm{E}-06-9.78 \mathrm{E}-05$ & $49.5-81.4$ \\
\hline …............... & 1 & 0.69 & 4.69 & 846 & $0.0-60.0$ & $5.00 \mathrm{E}-01-9.97 \mathrm{E}+00$ & $4.47 \mathrm{E}+01-4.46 \mathrm{E}+03$ & $0-1.04 \mathrm{E}-03$ & $18.2-81.4$ \\
\hline $11 \ldots$ & 1 & 0.17 & 5.17 & 879 & $5.2-50.2$ & $2.13 \mathrm{E}+00-9.92 \mathrm{E}+00$ & $2.12 \mathrm{E}+02-5.16 \mathrm{E}+03$ & $0-2.18 \mathrm{E}-03$ & $18.2-81.4$ \\
\hline $12^{\mathrm{d}} .$. & 1 & 6.62 & 36.13 & 10 & $38.8-41.5$ & $1.36 \mathrm{E}+01-1.39 \mathrm{E}+01$ & $6.92 \mathrm{E}+03-9.39 \mathrm{E}+03$ & $5.91 \mathrm{E}-04-9.96 \mathrm{E}-04$ & 56.6 \\
\hline $13 \ldots$ & 1 & 3.11 & 8.11 & 200 & $7.5-60.0$ & $9.65 \mathrm{E}-01-4.25 \mathrm{E}+00$ & $1.12 \mathrm{E}+01-1.49 \mathrm{E}+02$ & $0-1.01 \mathrm{E}-04$ & $18.2-87.1$ \\
\hline $14 \ldots$ & 1 & 1.57 & 6.36 & 31 & $0.0-18.7$ & $4.33 \mathrm{E}-01-2.70 \mathrm{E}+00$ & $5.32 \mathrm{E}+00-3.16 \mathrm{E}+01$ & $7.22 \mathrm{E}-06-1.10 \mathrm{E}-04$ & $41.4-81.4$ \\
\hline 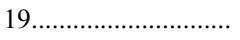 & 1 & 1.19 & 6.18 & 209 & $19.4-60.0$ & $2.65 \mathrm{E}+00-4.46 \mathrm{E}+00$ & $2.89 \mathrm{E}+01-3.14 \mathrm{E}-$ & $0-2.82 \mathrm{E}-08$ & $18.2-81.4$ \\
\hline $20 \ldots$ & 1 & 4.89 & 11.30 & 14 & $0.0-7.1$ & $6.60 \mathrm{E}-01-1.54 \mathrm{E}+00$ & $4.82 \mathrm{E}+00-1.44 \mathrm{E}+01$ & $0-8.48 \mathrm{E}-06$ & $18.2-81.4$ \\
\hline $21 \ldots \ldots \ldots \ldots \ldots \ldots \ldots \ldots$ & 1 & 18.30 & 26.23 & 14 & 9.1 & 6.14 & $+02-1$ & 0 & $18.2-87.1$ \\
\hline $22 \ldots \ldots \ldots \ldots \ldots \ldots$ & 1 & 0.01 & 4.01 & 425 & -60.0 & $4.18 \mathrm{E}-01-4$. & $5.00 \mathrm{E}+00-3$ & $0-1.09 \mathrm{E}-04$ & $18.2-81.4$ \\
\hline $23 \ldots \ldots \ldots \ldots \ldots \ldots$ & 1 & 1.72 & 8.72 & 165 & $0.0-21.0$ & $1.00 \mathrm{E}+00-3.83 \mathrm{E}+00$ & $1.04 \mathrm{E}+01-5.3$ & $0-1.88 \mathrm{E}-05$ & $18.2-81.4$ \\
\hline .................... & 1 & 0.62 & 5.62 & 1070 & $0.0-45.6$ & $1.80 \mathrm{E}-01-3.73 \mathrm{E}+00$ & $1.44 \mathrm{E}+00-5.09 \mathrm{E}+01$ & $0-2.99 \mathrm{E}-05$ & $18.2-81.4$ \\
\hline …................ & 1 & 0.24 & 4.23 & 22 & $6.7-10.5$ & $1.89 \mathrm{E}+00-2.77 \mathrm{E}+00$ & $1.25 \mathrm{E}+01-7.58 \mathrm{E}+01$ & 0 & $18.2-87.1$ \\
\hline$\ldots \ldots \ldots \ldots \ldots$ & 1 & 0.02 & 3.98 & 221 & $18.6-54.0$ & $2.05 \mathrm{E}+00-3.99 \mathrm{E}+00$ & $1.67 \mathrm{E}+01-2.05 \mathrm{E}+02$ & 0 & $18.2-87.1$ \\
\hline $27 \ldots \ldots \ldots \ldots \ldots \ldots \ldots$ & 1 & 0.39 & 5.38 & 293 & $0.0-52.3$ & $4.14 \mathrm{E}-01-5.03 \mathrm{E}+00$ & $5.29 \mathrm{E}+00-1.12 \mathrm{E}+02$ & $2.95 \mathrm{E}-06-3.31 \mathrm{E}-04$ & $31.8-81.4$ \\
\hline $28 \ldots \ldots \ldots \ldots \ldots \ldots \ldots$ & 1 & 3.85 & 11.84 & 98 & $3.6-14.5$ & $7.91 \mathrm{E}-01-3.64 \mathrm{E}+00$ & $6.74 \mathrm{E}+00-3.20 \mathrm{E}+01$ & $0-1.86 \mathrm{E}-05$ & $41.4-81.4$ \\
\hline 29 & 1 & 17.39 & 21.98 & 7 & $3.9-17.8$ & $4.84 \mathrm{E}+00-6.80 \mathrm{E}+00$ & $1.69 \mathrm{E}+02-3.42 \mathrm{E}+02$ & $6.03 \mathrm{E}-05-2.10 \mathrm{E}-04$ & $41.4-75.5$ \\
\hline $30 \ldots \ldots \ldots \ldots \ldots$ & 1 & 3.54 & 8.54 & 186 & $1.4-60.0$ & $1.49 \mathrm{E}-01-4.71 \mathrm{E}+00$ & $1.99 \mathrm{E}+00-2.92 \mathrm{E}+02$ & $0-1.71 \mathrm{E}-04$ & $18.2-81.4$ \\
\hline $31 \ldots \ldots \ldots \ldots \ldots \ldots$ & 2 & 39.45 & 51.32 & 19 & $7.4-40.5$ & $7.51 \mathrm{E}+00-1.34 \mathrm{E}+01$ & $1.02 \mathrm{E}+03-3.84 \mathrm{E}+03$ & $2.67 \mathrm{E}-04-2.21 \mathrm{E}-03$ & $31.8-87.1$ \\
\hline
\end{tabular}

a The range of values reported here for each YSO parameter has a 95\% probability, based on the probability distribution of best fits, of containing the actual value of that parameter for each source.

${ }^{b}$ Fits having $\chi^{2} \leq \chi_{\max }^{2}$ are used in computing the probability distribution of YSO parameters. For each source, $\chi_{\max }^{2}-\chi_{\min }^{2}=\Delta N_{\text {data }}$, where $N_{\text {data }}$ is the number of flux data points used in the fit and $\Delta=1$ for Fit $=1$ and $\Delta=3$ for Fit $=2$.

c For models with $\dot{M}_{\text {env }}<10^{-9} M_{\odot} \mathrm{yr}^{-1}$, the envelope accretion rate is considered to be zero.

d For this source, the IR counterpart to G34 MM, we report the probability distribution of parameters from the 10 best fits.

evolved than source 12 . In very young sources, particularly those with $M_{\star}>2 M_{\odot}$, high accretion rates create a cool, distended photosphere that does not emit strongly enough in the UV to excite the PAH emission features present in the IRAC [3.6], [5.8], and [8.0] bands. In more evolved sources, however, PAH emission can hide the contrast between [4.5] and the other three bands. Alternatively, as discussed above, both sources 10 and 11 suffer from confusion with the $\mathrm{UC} \mathrm{H}$ II region, which emits strongly in all four bands, and this could also mask moderate enhancements due to $\mathrm{CO}$ band head emission.

As a final note, three sources show marginal excess at $4.5 \mu \mathrm{m}$ relative to bands [5.8] and [8.0]: source 7 in region $C$ and sources 20 and 26 in region N. These sources do not appear to have strong emission at $24 \mu \mathrm{m}$, so they may be a different type of source than those with strong 4.5 and $24 \mu \mathrm{m}$ excesses.

\subsection{Timescale for Massive Star Formation}

There are two cloud cores in the central region of the filament: one more massive core in the south surrounded by a more diffuse molecular cloud that contains the $\mathrm{UC} \mathrm{H}$ II region and one that is more compact containing source 12/G34.4 MM. The cores are separated by about $0.75 \mathrm{pc}$. Each cloud core is producing a small cluster of massive flows that look similar and are of similar ages, although the northern outflows associated with G34.4 MM have about one-third the mass of the southern flows.

Due to the shorter formation timescales $\left(\lesssim 10^{5} \mathrm{yr}\right)$, massive (proto-) stars begin to burn hydrogen even while they continue to accrete material. The generation of a UC H II region identifies which sources have reached the main sequence and begun hydrogen burning, while the detection of a molecular outflow traced to the source identifies whether it is still accreting (and hence still powering an outflow). However, the addition of material onto the stellar surface implies that the source will move up the main sequence. In this sense, it is still a "protostar " because it has not reached its final mass.

The central source in the G34.4 UC H II region (source 11) has a spectral type of $\mathrm{B} 0.5$, while the spectral type of source 12/G34.4 MM is probably closer to a B2 star (this work; SNB04). High-velocity CO emission can be traced to both sources, suggesting that they are still accreting even though they have reached the main sequence.

The characteristic timescale of the outflow/infall phase from early B stars is roughly a few $\times 10^{5} \mathrm{yr}$ (Richer et al. 2000), while the evolutionary timescale for a B0.5 star to reach the main sequence is roughly $10^{5} \mathrm{yr}$. Since ionized gas is seen toward both cores and the outflow characteristics are similar, it seems reasonable 

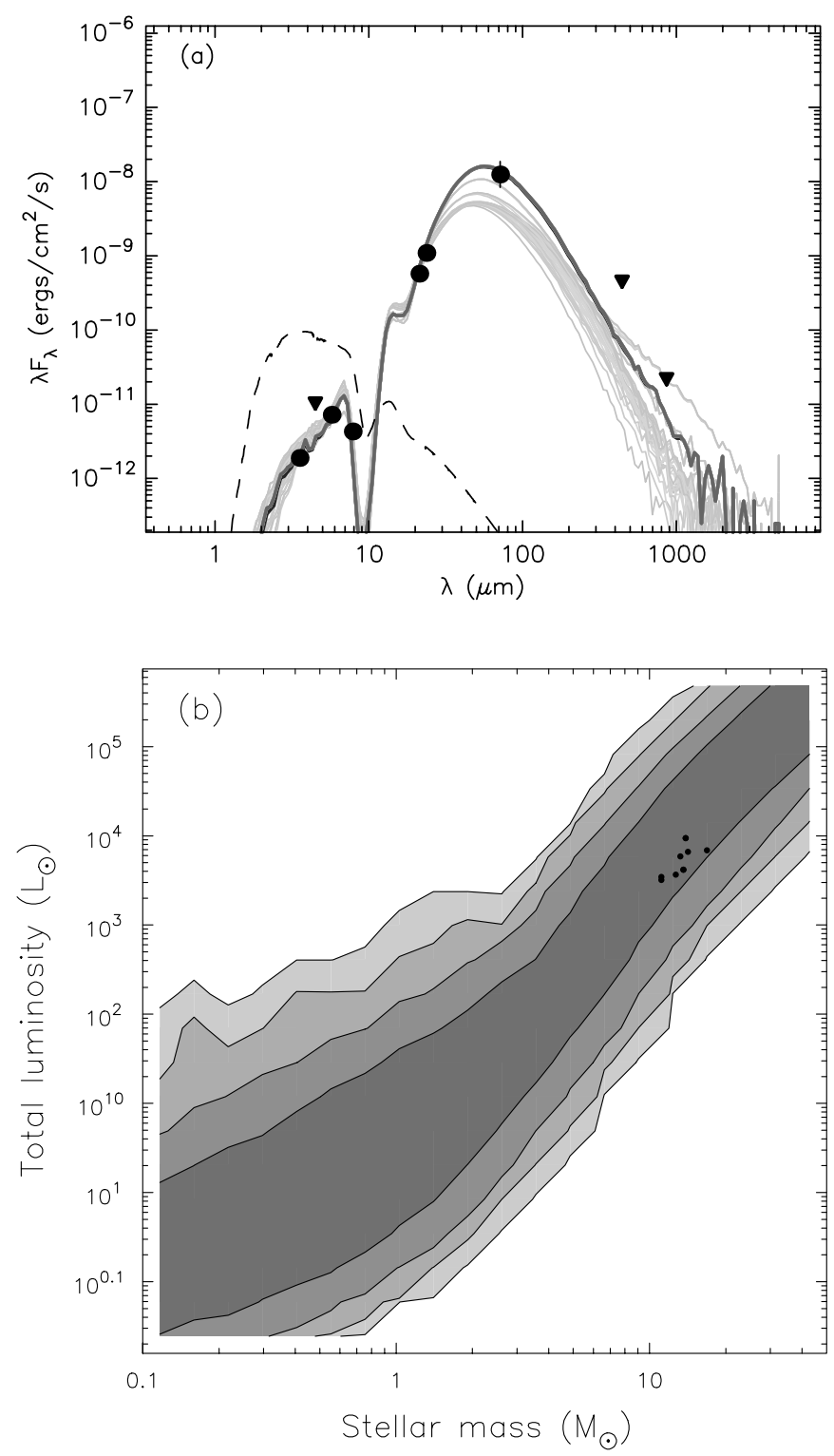

FIG. 11.-(a) Ten best YSO SED model fits to the IR fluxes of source $12 / \mathrm{G} 34.4 \mathrm{MM}$. The fitting assumes that the mid-IR flux from the millimeter core is dominated by a single source. The [4.5] upper limit (triangles) was used because the source appears to have excess flux in this bandpass due to molecular emission associated with outflow activity that has not been incorporated into the YSO models. The thickest line represents the best fit to the data, and light gray lines represent the SED observed through different apertures, from the smallest (IRAC) to largest $(M S X 21.3 \mu \mathrm{m})$. The dashed line shows the photospheric flux from the best-fit YSO model as it would appear if suffering from interstellar extinction only in the absence of circumstellar material. (b) Masses and luminosities of the best-fit models. The shaded contour regions represent the logarithmic density of models in the mass-luminosity parameter space. The darkest gray represents a model density 1000 times higher than that of the lightest gray. The white region beyond the lowest contour shows the part of parameter space that lies beyond the edge of the model grid.

to assume that they began forming massive stars at approximately the same time, about $10^{5}$ yr ago.

SNB04 detected a lower mass population of this cluster (e.g., a few solar masses or less) at near-IR wavelengths (see their Fig. 6). Their detected cluster population was more distributed, less embedded, and less massive than those detected in this work. They found that this subpopulation is less than $3 \mathrm{Myr}$ old but probably more than 0.3 Myr old.

There are numerous problems associated with these age estimates, and they could be in error easily by a factor of a few. How- ever, let us take them at face value for now. SNB04 discovered a distributed population of low- to intermediate-mass stars that may have formed a few Myr ago. The more massive stars detected in this work are more tightly associated with the dense molecular gas (as expected) and appear to be somewhat younger, more like $10^{5} \mathrm{yr}$ old. This suggests that the star formation in this cold, dark cloud may have formed in two stages: first lower mass stars began to form then, a few Myr later, the more massive, early B stars began to form.

Such a situation is predicted when clouds have magnetic fields that are stronger than needed to provide support against gravitational collapse and the core mass to magnetic flux ratio $(M / \Phi)$ is subcritical. Thus, low-mass stars would form in originally highly magnetically subcritical clouds, with ambipolar diffusion leading to core formation, quasi-static contraction of the cores, and eventually, massive star formation (see, e.g., the review by WardThompson et al. 2007 and references therein). If this scenario is correct, then the cold, dark filament associated with IRAS 18507 may provide an example of a cloud with delayed massive star formation due to an initially high magnetic field and subcritical $M / \Phi$.

\subsection{Star Formation Efficiency}

The set of best-fit SED models for YSO candidates can be used to construct a $\chi^{2}$-weighted probability distribution of the best-fit model parameters. We sum the mass probability distributions for each source to construct mass functions for all well-fit YSOs $($ Fit $=1)$ in Tables 3 and 4 . The total mass of the GLIMPSEdetected YSOs in this IR-dark cloud is then $127 \pm 27 M_{\odot}$.

The total stellar mass of the central (sub-) cluster containing G34.4 MM and the UC H II region is $48 \pm 20 M_{\odot}$ (or 38\% of the total detected stellar mass). About $14 M_{\odot}$ are in source 12/G34.4 MM, $10 M_{\odot}$ are in source 31 (the yellow source near G34.4 MM in Fig. 10), and the remaining $24 M_{\odot}$ of stellar mass resides in the central star producing the $\mathrm{UC} \mathrm{H}$ II region and its surrounding cluster. These rough stellar mass estimates are incomplete for $M_{\star}<3 M_{\odot}$. They also do not include the additional $20 \mathrm{YSO}$ candidates that were detected at $24 \mu \mathrm{m}$ but in fewer than three IRAC bands. Thus, the stellar mass estimate should be considered a lower limit.

Using the initial mass function (IMF) of Kroupa (2001; where the IMF slope follows a Salpeter IMF for $M_{\star}>1 M_{\odot}$ and it becomes shallower for $0.1 M_{\odot}<M_{\star}<1 M_{\odot}$ ), one can estimate the expected stellar mass for $M_{\star}<3 M_{\odot}$. Assuming $127 \pm 27 M_{\odot}$ of mass in stars more massive than $3 M_{\odot}$, roughly $413 \pm 103 M_{\odot}$ of mass is expected to be in the form of stars less massive than $3 M_{\odot}$. Thus, the total stellar mass content of this cloud is expected to be $\sim 540 \pm 130 M_{\odot}$.

This stellar mass estimate is a little more than half of the derived mass of the ammonia hot core of $1000 M_{\odot}$ surrounding the $\mathrm{UCH}_{\text {II }}$ region (Miralles et al. 1994), and it is about 7\% of the cloud mass of $7500 M_{\odot}$ derived from $1.2 \mathrm{~mm}$ continuum emission (Rathborne et al. 2005). The total star formation efficiency is then $M_{\star} /\left(M_{\text {cld }}+\right.$ $\left.M_{\star}\right)=540 / 8040 \sim 0.07$ or $7 \%$. This should be considered an upper limit, because $1.2 \mathrm{~mm}$ continuum emission traces warm dust (typically $30-50 \mathrm{~K}$ ) which is heated predominantly by massive stars-it does not effectively trace the cold gas and dust (5-10 K) which may represent the bulk of the total cloud mass.

We can also obtain an estimate of the massive and intermediatemass star formation efficiency in the warm gas traced by $1.2 \mathrm{~mm}$ continuum emission and in the densest part of the hot core. Assuming a warm cloud mass of $7500 M_{\odot}$ and a total mass of stars with $M_{\star}>3 M_{\odot}=127 M_{\odot}$, the massive star formation efficiency is roughly $2 \%$. 

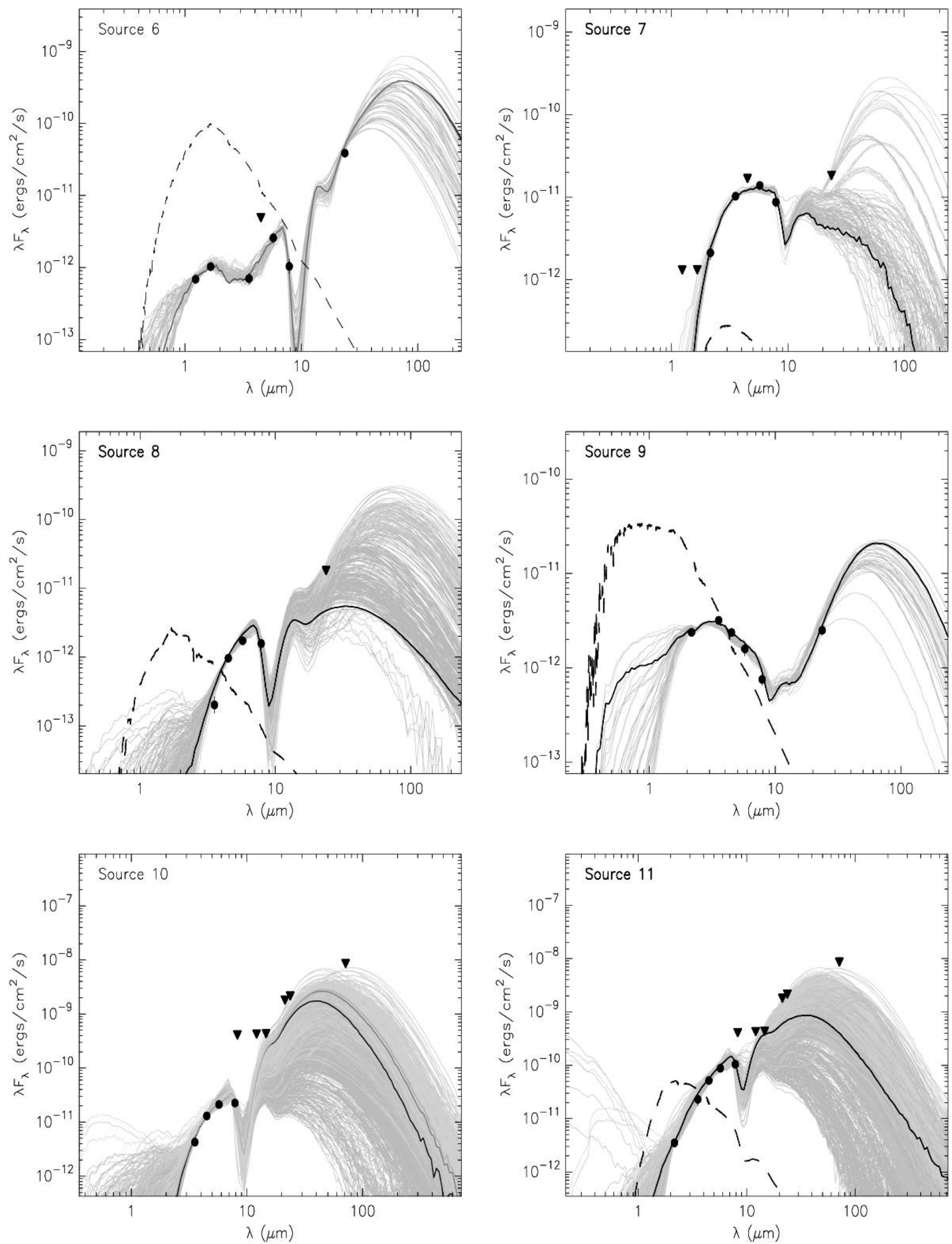

FIG. 12.- (a) Same as Fig. 11a, but for the other six well-fit YSOs in region C. All fits used to compute the model parameter ranges in Table 4 are plotted. Sources 10 and

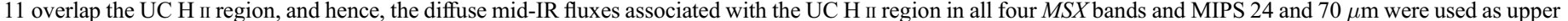
limits to the fits. (b) Same as Fig. 11b, but for the six well-fit YSOs plotted in $(a)$. 

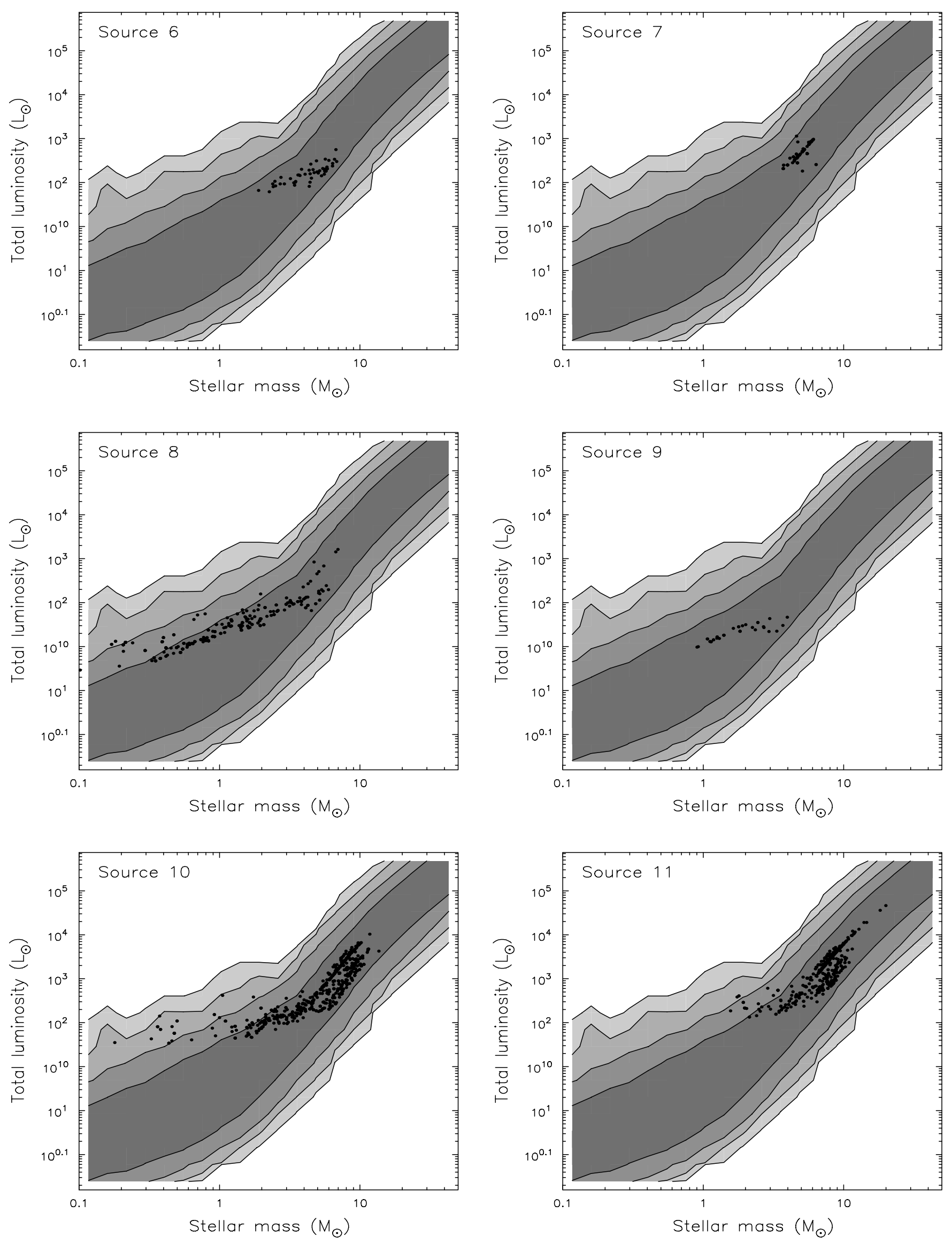


\subsection{Can the Outflows Destabilize the Cloud Cores?}

\subsubsection{The G34.4 Cloud Core Surrounding the UC H II Region}

Miralles et al. (1994) estimated the mass of the G34.4 cloud core surrounding the UC $\mathrm{H}$ II region to be $\sim 1000 M_{\odot}$ based on $\mathrm{NH}_{3}$ observations. They only detected emission near the UC H II region, not near the $\mathrm{G} 34.4 \mathrm{MM}$ core. The $\mathrm{C}^{18} \mathrm{O}$ mass estimate of $693 M_{\odot}$ compares reasonably well with that of $\mathrm{NH}_{3}$ given the uncertainties inherent with both estimates and the fact that our $\mathrm{C}^{18} \mathrm{O}$ map is missing extended emission.

If we take the mass of the G34.4 core surrounding the UC H II region to be $M_{\text {cld }}=1000 M_{\odot}$, we find that the core has about 9 times more mass than in the high-velocity molecular outflows. The gravitational binding energy of the cloud can be calculated from $G M_{\text {cld }}^{2} / c_{1} r$, where $c_{1}$ is a constant which depends on the mass distribution $\left(c_{1}=1\right.$ for $\left.\rho \propto r^{-2}\right)$. We find that the gravitational binding energy is $2.4 \times 10^{47} \mathrm{ergs}$. Roughly $11 \%$ of the molecular cloud core is participating in the outflow, and the combined outflow energy is roughly one-third the gravitational binding energy of the cloud. The G34.4 outflows near the UC H II region are injecting a significant amount of mechanical energy into the cloud core and may help prevent further collapse of the cloud. However, they are not likely to totally disrupt the cloud. As long as cloud material remains, this leaves the door open for possible future episodes of star formation.

\subsubsection{The Compact G34.4 MM Cloud Core}

SNB04 estimate the mass of the G34.4 MM core based on $2.6 \mathrm{~mm}$ thermal dust emission to be between 150 and $650 M_{\odot}$ with $240 M_{\odot}$ being the value derived for an opacity index $\beta$ of 1.5 and a temperature of $50 \mathrm{~K}$. Our estimated mass based on $\mathrm{C}^{18} \mathrm{O}$ emission is significantly less than this $\left(\sim 75 M_{\odot}\right)$, suggesting that our $\mathrm{C}^{18} \mathrm{O}$ maps are missing extended emission or the assumptions used to estimate the mass associated with warm dust emission in the G34.4 MM core were not correct. Thus, for example, if $\beta=0.7$ and $T_{d}=70 \mathrm{~K}$, then the mass of the millimeter core would be $\sim 75 M_{\odot}$, which is consistent with the mass derived from $\mathrm{C}^{18} \mathrm{O}$. Such a low opacity index and high temperature could be caused by the presence of strong shocks through most of the core mass, which would prevent large grains from forming and maintain the bulk of the gas at higher temperatures. Indeed, this could be possible given the small size of the core $(0.2 \mathrm{pc})$ and the fact that two outflows with a combined mass of nearly $35 M_{\odot}$ are blasting their way out of the core.

If we assume a mass of $75-240 M_{\odot}$ for the G34.4 MM core, as derived from the $\mathrm{C}^{18} \mathrm{O}$ and $2.6 \mathrm{~mm}$ continuum observations, then the gravitational binding energy of the cloud is $5.6 \times 10^{45}$ $5.7 \times 10^{46}$ ergs. The kinetic energy of the outflowing gas is between 1.2 and nearly 9 times the gravitational binding energy of the cloud core. Thus, the G34.4 MM flows appear to have more energy relative to the core and are more likely to disrupt the compact core. Therefore, we may be seeing the first and only episode of massive star formation in this compact region.

\section{SUMMARY}

We have observed the central region of the IR-dark cloud associated with IRAS $18507+0121$ in $\mathrm{CO}(J=1-0),{ }^{13} \mathrm{CO}(J=1-0)$, and $\mathrm{C}^{18} \mathrm{O}(J=1-0)$ with the Owens Valley Radio Observatory at $\sim 4^{\prime \prime}$ resolution. Five massive outflows from two cloud cores were discovered. A total of $146 M_{\odot}$ of material is participating in the combined outflows and injecting $\sim 1.3 \times 10^{45}$ ergs of kinetic energy. We also used archived Spitzer data from the GLIMPSE survey to gain an understanding of the stellar content of the cluster and the entire IR-dark filament. Modeling of the SEDs of GLIMPSE sources identified 31 young stellar objects in the filament with a combined stellar mass of $\sim 127 \pm 27 M_{\odot}$. An additional 22 sources were identified as probable cluster members based on the presence of strong $24 \mu \mathrm{m}$ emission. The total star formation efficiency in the G34.4 cloud filament is estimated to be about 7\%, while the massive and intermediate-mass star formation efficiency in the entire cloud filament is estimated to be roughly $2 \%$.

A qualitative evaluation of the outflow characteristics and presence of ionized gas in the central region of the IR-dark cloud filament suggests that the early B stars currently undergoing outflow and accretion began forming stars at approximately the same time, about $10^{5} \mathrm{yr}$ ago. A population of lower mass stars were detected by SNB04 that appear to have an age of about a few Myr. This dual population suggests that stars in the IRAS 18507 cloud may have formed in two stages: first lower mass stars formed and then, a few Myr later, the more massive, early B stars began to form. Such a situation can occur in magnetically subcritical clouds in which slow, ambipolar diffusion leads to the delayed onset of core contraction and eventual massive star formation.

A comparison of the gravitational binding energy with the outflow kinetic energy in the two cloud cores studied in molecular lines suggests that the compact core containing G34.4 MM is being destroyed by its molecular outflows. The outflows have as much or more kinetic energy than the gravitational binding energy of the cloud core. Thus, we may be seeing the first and only episode of massive star formation in this compact region.

In contrast, the $690 M_{\odot}$ core surrounding the G34.4 UC H II region has twice the gravitational binding energy as the combined outflow kinetic energy. Thus, although the outflows are injecting a significant amount of mechanical energy into the cloud core and may help prevent further collapse of the cloud, they are not likely to totally disrupt the cloud. As long as cloud material remains, this leaves the door open for possible future episodes of star formation.

D. S. S. would like to thank Allison Sills for useful discussions on properties of young clusters. Research at the Owens Valley Radio Observatory is supported by the National Science Foundation through NSF grant AST 96-13717. Star formation research at Owens Valley is also supported by NASA's Origins of Solar Systems program, grant NAGW-4030, and the Norris Planetary Origins Project. L. B. acknowledges support from the Chilean Center for Astrophysics FONDAP grant 15010003. Support for GLIMPSE, part of the Spitzer Space Telescope Legacy Science Program, was provided by NASA through contracts 1224653 (University of Wisconsin at Madison) and 1224988 (Space Science Institute). B. W. gratefully acknowledges support from the NASA Astrophysics Theory Program (NNG 05-GH35G) and the Spitzer Space Telescope Theoretical Research Program (subcontract 1290701).
Benjamin, R. A., et al. 2003, PASP, 115, 953

Bronfman, L., Nyman, L., \& May, J. 1996, A\&AS, 115, 81

Brott, I., \& Hauschildt, P. H. 2005, in The Three-Dimensional Universe with Gaia, ed. C. Turon, K. S. O'Flaherty, \& M. A. C. Perryman (ESA SP-576; Noordwijk: ESA), 565

\section{REFERENCES}

Cabrit, S., \& Bertout, C. 1990, ApJ, 348, 530

Carey, S. J., et al. 2005, BAAS Abstr., 207, 6333

Carral, P., \& Welch, W. J. 1992, ApJ, 385, 244

Davis, C. J., Kumar, M. S. N., Sandell, G., Froebrich, D., Smith, M. D., \& Currie, M. J. 2007, MNRAS, 374, 29 
Edris, K. A., Fuller, G. A., \& Cohen, R. J. 2007, A\&A, 465, 865

Faúndez, S., Bronfman, L., Garay, G., Chini, R., Nyman, L.-A., \& May, J. 2004, A\&A, 426, 97

Fazio, G. G., et al. 2004, ApJS, 154, 10

Kroupa, P. 2001, MNRAS, 322, 231

Kurucz, R. L. 1993, Kurucz CD-ROM 13, ATLAS 9 Stellar Atmosphere Programs and $2 \mathrm{~km} / \mathrm{s}$ Grid (Cambridge: $\mathrm{SAO}$ )

Miralles, M. P., Rodriguez, L. F., \& Scalise, E. 1994, ApJS, 92, 173

Molinari, S., Brand, J., Cesaroni, R., \& Palla, F. 1996, A\&A, 308, 573

Molinari, S., Brand, J., Cesaroni, R., Palla, F., \& Palumbo, G. G. C. 1998, A\&A, 336, 339

Noriega-Crespo, A., et al. 2004, ApJS, 154, 352

Palla, F., Brand, J., Comoretto, G., Felli, M., \& Cesaroni, R. 1991, A\&A, 246, 249

Price, S. D., Egan, M. P., Carey, S. J., Mizuno, D. R., \& Kuchar, A. 2001, AJ, 121,2819

Qiu, K., Zhang, Q., Gutermuth, R. A., Megath, S. T., Beuther, H., Sridharan, T. K., Shepherd, D. S., Testi, L., \& De Pree, C. G. 2007, BAAS Abstr., 209, 3005

Ramesh, B., Bronfman, L., \& Deguchi, S. 1997, PASJ, 49, 307

Rathborne, J. M., Jackson, J. M., Chambers, E. T., Simon, R., Shipman, R., \& Frieswijk, W. 2005, ApJ, 630, L181

Richer, J. S., Shepherd, D. S., Cabrit, S., Bachiller, R., \& Churchwell, E. 2000, in Protostars and Planets IV, ed. V. Mannings, A. Boss, \& S. Russell (Tucson: Univ. Arizona Press), 867

Rieke, G. H., et. al. 2004, Proc. SPIE, 5487, 50

Robitaille, T. P., Whitney, B. A., Indebetouw, R., \& Wood, K. 2007, ApJS, 169, 328
Robitaille, T. P., Whitney, B. A., Indebetouw, R., Wood, K., \& Denzmore, P. 2006, ApJS, 167, 256

Sault, R. J., Teuben, P. J., \& Wright, M. C. H. 1995, in ASP Conf. Ser. 77, Astronomical Data Analysis Software and Systems IV, ed. R. A. Shaw, H. E. Payne, \& J. J. E. Hayes (San Francisco: ASP), 433

Scalise, E., Jr., Rodríguez, L. F., \& Mendoza-Torres, E. 1989, A\&A, 221, 105 Schutte, A. J., van der Walt, D. J., Gaylard, M. J., \& MacLeod, G. C. 1993, MNRAS, 261, 783

Scoville, N. Z., Carlstrom, J. E., Chandler, C. J., Phillips, J. A., Scott, S. L., Tilanus, R. P. J., \& Wang, Z. 1993, PASP, 105, 1482

Scoville, N. Z., Sargent, A. I., Sanders, D. B., Claussen, M. J., Masson, C. R., Lo, K. Y., \& Phillips, T. G. 1986, ApJ, 303, 416

Shepherd, D. S., Nürnberger, D. E. A., \& Bronfman, L. 2004, ApJ, 602, 850 (SNB04)

Skrutskie, M. F., et al. 2006, AJ, 131, 1163

Smith, H. A., Hora, J. L., Marengo, M., \& Pipher, J. L. 2006, ApJ, 645, 1264 Stetson, P. B. 1987, PASP, 99, 191

Szymczak, M., Hrynek, G., \& Kus, A. J. 2000, A\&AS, 143, 269

Wang, Y., Zhang, Q., Rathborne, J. M., Jackson, J., \& Wu, Y. 2006, ApJ, 651, L125

Ward-Thompson, D., André, P., Crutcher, R., Johnstone, D., Onishi, T., \& Wilson, C. 2007, in Protostars and Planets V, ed. B. Reipurth, D. Jewitt, \& K. Keil (Tucson: Univ. Arizona Press), 33

Wilson, T. L., \& Rood, R. T. 1994, ARA\&A, 32, 191

Wood, D. O. S., \& Churchwell, E. 1989, ApJS, 69, 831

Zhang, Q., Hunter, T. R., Brand, J., Sridharan, T. K., Cesaroni, R., Molinari, S., Wang, J., \& Kramer, M. 2005, ApJ, 625, 864 